\title{
Diversity, Variability, and Suboesophageal Connectivity of Antennal Lobe Neurons in D. melanogaster Larvae
}

\author{
A.S. Thum, ${ }^{1 *}$ B. Leisibach, ${ }^{1}$ N. Gendre, ${ }^{1}$ M. Selcho, ${ }^{1,2}$ and R.F. Stocker ${ }^{1}$ \\ 'Department of Biology, University of Fribourg, 1700 Fribourg, Switzerland \\ ${ }^{2}$ Department of Biology, Philipps-University Marburg, 35032 Marburg, Germany
}

\section{ABSTRACT}

Whereas the "vertical" elements of the insect olfactory pathway, the olfactory receptor neurons and the projection neurons, have been studied in great detail, local interneurons providing "horizontal" connections in the antennal lobe were ignored for a long time. Recent studies in adult Drosophila demonstrate diverse roles for these neurons in the integration of odor information, consistent with the identification of a large variety of anatomical and neurochemical subtypes. Here we focus on the larval olfactory circuit of Drosophila, which is much reduced in terms of cell numbers. We show that the horizontal connectivity in the larval antennal lobe differs largely from its adult counterpart. Only one of the five anatomical types of neurons we describe is restricted to the antennal lobe and therefore fits the defi-
\end{abstract}

nition of a local interneuron. Interestingly, the four remaining subtypes innervate both the antennal lobe and the suboesophageal ganglion. In the latter, they may overlap with primary gustatory terminals and with arborizations of hugin cells, which are involved in feeding control. This circuitry suggests special links between smell and taste, which may reflect the chemosensory constraints of a crawling and burrowing lifestyle. We also demonstrate that many of the neurons we describe exhibit highly variable trajectories and arborizations, especially in the suboesophageal ganglion. Together with reports from adult Drosophila, these data suggest that wiring variability may be another principle of insect brain organization, in parallel with stereotypy.

INDEXING TERMS: Drosophila; larvae; olfactory system; interneurons

The olfactory pathway of Drosophila and other insects typically comprises three major cellular elements: olfactory receptor neurons (ORNs), which transmit odor signals from the periphery to the antennal lobe (AL); local interneurons, which provide horizontal connections inside the $\mathrm{AL}$; and projection neurons (PNs), which pass the processed information from the AL on to higher olfactory centers, i.e., the mushroom body and the lateral horn. Given the genetic tractability of the fly brain and its simplicity in terms of cell numbers, the molecular circuitry of ORNs and PNs has been dissected to a remarkable degree (for review, see Vosshall and Stocker, 2007). In contrast, local interneurons have only recently become a focus of interest. The dominant class of local interneurons, which are $\gamma$-aminobutyric acid (GABA)ergic and inhibitory, were reported to enhance neural representations of discrete odors (Wilson and Laurent, 2005), to be involved in olfactory gain control (Olsen and Wilson, 2008), and/or to mediate oscillatory synchronization of PNs (Tanaka et al., 2009). A newly identified class of cho- linergic local interneurons was shown to establish excitatory connections, whose major purpose may be to boost PN signals (Olsen et al., 2007; Root et al., 2007; Shang et al., 2007).

Local interneurons in Drosophila were initially considered to be anatomically rather uniform, innervating most if not all glomeruli of the AL. However, various other types displaying heterogeneous $\mathrm{AL}$ innervation have been described since 2005 (Wilson and Laurent, 2005; Das et al., 2008; Lai et al., 2008; Okada et al., 2009). A recent report demonstrated surprising cell-type diversity and

Grant sponsor: Swiss National Funds; Grant numbers: 31003A-105517 (to R.F.S), 31003 A-132812 (to A.S.T.), and PBFRP3-133515 (to M.S.) Grant sponsor: Swiss University Conference (to A.S.T.).

The first two authors contributed equally to this work.

${ }^{*}$ CORRESPONDENCE TO: Andreas S. Thum, Department of Biology, University of Fribourg, Chemin du Musée 10, 1700 Fribourg, Switzerland. E-mail: andreas.thum@unifr.ch 
wiring variability of these neurons in the adult AL (Chou et al., 2010), challenging the prevailing idea that insect brains are entirely or at least predominantly hardwired. Nevertheless, all types of newly identified neurons arborized exclusively in the AL, complying with the definition of local interneurons.

Here we focus on interneurons of the larval AL. The larval olfactory pathway has become a particularly attractive model system as it shares the basic design of the adult pathway but comprises only $21 \mathrm{ORNs}$ and not many more PNs (Python and Stocker, 2002a; Masuda-Nakagawa et al., 2005, 2009; Ramaekers et al., 2005).

We address two key questions: 1) are larval $\mathrm{AL}$ interneurons homologous to those identified in the adult, and 2) does their connectivity also reveal an unusually high degree of variability? Analyzing single-cell clones in three GAL4 driver lines allowed us to identify anatomically distinct subtypes of neurons in the larval AL that were neither ORNs nor PNs. Only one of them was a genuine local interneuron, whereas the remaining subtypes also innervated the SOG and-in one case-additionally a subregion of the mushroom body lobes. For most of the cell types reported from the adult AL (Das et al., 2008; Chou et al., 2010), we did not detect equivalents in the larval AL. Hence, the cellular composition of adult and larval ALs appears to be largely different. However, similar to the adult situation, we found a high degree of arborization variability in the larval neurons, in particular with respect to SOG patterns.

\section{MATERIALS AND METHODS}

\section{Fly strains and fly breeding}

For screening of GAL4 expression patterns, we crossed males of the GAL4 lines C739 (Yang et al., 1995), GH146 (Stocker et al., 1997; Heimbeck et al., 2001), GH298 (Stocker et al., 1997), H24 (Martin et al., 1998), HB4-93 and HB8-145 (both from U. Heberlein; Chou et al., 2010), Krasavietz-GAL4 (Shang et al., 2007), LCCH3 (J. Simpson; Chou et al., 2010), Nan-GAL4 (Kim et al., 2003), NP0385, NP1227, NP1612, NP2426, NP3056, and NP6277 (all from the NP Consortium, Japan), OK107 (Lee et al., 1999), and 189Y-GAL4; mb247-GAL80 (Osborne et al., 1997; Python and Stocker, 2002a; Krashes et al., 2007) with virgin females of the two reporter lines UASmCD8::GFP (Bloomington Stock Center, Bloomington, IN) or UAS-Cameleon 2.1 (UAS-Cam; Diegelmann et al., 2002). For inducing single-cell clones, males of $189 \mathrm{Y}$ GAL4; mb247-GAL80, NP2426-GAL4, NP3056-GAL4, or GH146-GAL4 were crossed with virgin females of the responder line hsFLP; CyO/SP; UAS >y+CD2>pCD8::GFP (WoNg et al., 2002).
To identify pre- and postsynaptic sites of neurons, males of 189Y-GAL4; mb247-GAL80, NP2426-GAL4, and NP3056-GAL4 were crossed with virgin females of the responder lines UAS-nsyb::GFP (Ito et al., 1998) and UASDscam 17.1::GFP (Wang et al., 2004a). Co-localization analysis of 189Y-GAL4; mb247-GAL80, NP2426-GAL4, and NP3056-GAL4 was done by crossing males of these three lines with either hug::YFP; UAS-CD2 or GR66a::GFP; UAS-CD2 virgin females (Bader et al., 2007; Colomb et al., 2007).

Flies were raised at either $18^{\circ} \mathrm{C}$ (for clone analysis) or $25^{\circ} \mathrm{C}$ (all other experiments) on standardized cornmeal medium containing yeast.

\section{Clone induction}

Clones were induced by activating FLP recombinase via a heat shock $\left(37^{\circ} \mathrm{C}\right)$ of $18-19$ minutes' duration, applied at variable times from 48 to 96 hours after egg laying. Larvae were then allowed to continue development at $18^{\circ} \mathrm{C}$.

\section{Dissection, immunohistochemistry, and confocal microscopy}

Feeding third instar larvae (L3) were dissected in phosphate-buffered saline (PBS; $\mathrm{pH}$ 7.2). The CNS was fixed for 25-30 minutes at room temperature (RT) in $3.7-4 \%$ paraformaldehyde (PA) in PBST (PBS containing 3\% Triton$X$ 100; Sigma-Aldrich, St. Louis, MO). Specimens were then rinsed $3 \times 15$ minutes and an extra 2 hours in PBST at RT and preincubated at RT with 3-5\% normal goat serum (NGS; Vector, Burlingame, CA) in PBST for 1-2 hours.

As primary antibodies we used for all stages rabbit or sheep polyclonal serum against green fluorescent protein (rabbit $\alpha$-GFP; Molecular Probes, Eugene, OR, cat. \#A6455; 1:1,000; sheep $\alpha$-GFP; Serotec, Bicester, UK, cat. \#4745-1051; 1:1,000) and mouse anti-Discs-Large PDZ2 domain ( $\alpha$-DLG; Developmental Studies Hybridoma Bank [DSHB], lowa City, IA, 4F3; IA; 1:20), the latter for visualizing the $A L$ neuropil. In experiments aimed at neurotransmitter identification, we used mouse anti-choline acetyltransferase ( $\alpha$-ChAT; DSHB, 4B1, IA; 1:200) in combination with rat anti-CD8 $(\alpha-C D 8$; Caltag, South San Francisco, CA, cat. \#RM2200; 1:200), rabbit anti-GABA ( $\alpha$-GABA; Sigma, St. Louis, MO, cat. \#A2052, 1:500), or sheep $\alpha$-GFP (see above). For co-localization with gustatory and hugin-positive neurons, we used rabbit $\alpha$-GFP (see above) and mouse anti-CD2 (Serotec, cat. \#MCA154R; 1:200).

All antibodies were diluted in 3\% NGS in PBST. After overnight incubation at $4{ }^{\circ} \mathrm{C}$ with the primary antibodies, brains were rinsed $3 \times 15$ minutes and 2 hours in PBST before being incubated for another night with secondary 
antibodies at $4{ }^{\circ} \mathrm{C}$. As secondary antibodies (all from Molecular Probes; diluted 1:200 in PBST containing 3\% NGS), we used goat anti-rabbit IgG Alexa Fluor 488 (cat. \#A11008) and goat anti-mouse IgG Alexa Fluor 647 (cat. \#A21235). For neurotransmitter identification, we additionally applied goat anti-rat IgG Alexa Fluor 488 (cat. \#A11006), donkey anti-sheep IgG Alexa Fluor 488 (cat. \#A11015), and goat anti-rabbit IgG Alexa Fluor 568 (cat. \#A11011). For co-localization analysis we used goat anti-rabbit IgG Alexa Fluor 488 (see above) and goat antimouse IgG Alexa Fluor 647 (see above). After four to six rinses of 15 minutes in PBS, preparations were mounted in $50 \%$ glycerol in PBS or Vectashield (Vector) and viewed on a Leica TCS SP5 confocal microscope.

Optical sections were taken at intervals of $1 \mu \mathrm{m}$ with $10 \times 20 \times$, or $63 \times$ glycerol objectives. Image analysis was performed with Image-J $(\mathrm{NIH})$ software, and data were further processed with Photoshop (Adobe Systems, San Jose, CA). Curves (input to output options) were readjusted for each color independently but always on the whole picture by using Image-J. The intensity of nonspecific background staining was lowered by using the "dust and scratches" filter with Photoshop.

\section{Antibody characterization Anti-GFP}

Rabbit anti-GFP and sheep anti-GFP are regularly used for studies on Drosophila and zebrafish, (Thum et al., 2006; Collins et al., 2010). They recognize the ectopically expressed GFP protein, as demonstrated by the absence of labeling in wild-type brains respectively (data not shown).

\section{Anti-DLG}

Anti-Discs large (DLG; antibody 4F3 from DSHB) labels larval neuromuscular junctions (NMJs) in a pattern (Parnas et al., 2001) that is similar to that of a polyclonal anti-DLG widely used to label the Drosophila larval NMJ; mutant dlg larvae exhibit altered expression of the latter and striking changes in NMJ structure (Lahey et al., 1994).

\section{Anti-ChATB1}

DChAT (Drosophila choline acetyltransferase) is the synthetic enzyme for acetylcholine and thus a marker for neurons with a cholinergic phenotype. The antibody was shown to react with a single band at a position of approximately $80 \mathrm{kDa}$ in crude fly head samples (Takagawa and Salvaterra, 1996).

\section{Anti-CD8 $\propto$}

Rat anti-mouse $\operatorname{CD} 8 \alpha$-subunit antibody recognizes specifically the ectopically expressed CD8 protein in Drosophila as demonstrated in a study focusing on the adult mushroom body (Tanaka et al., 2008). No labeling was seen in our preparations when rat anti-mouse CD $8 \alpha$ antibody was used as the primary antibody for the larval brains unless the GAL4 drove expression of mCD8::GFP.

\section{Anti-GABA}

To detect the presence of GABA immunoreactivity, we used a polyclonal antibody from rabbit. Rabbit anti-GABA shows positive binding with GABA in a dot blot assay and negative binding with bovine serum albumin (BSA; Sigma data sheet). The GABA antibody has been extensively used in amphibians (Bachy and Retaux, 2006; Moreno and Gonzalez, 2007) and was also used in a recent study in adult Drosophila to describe the morphology of GABAimmunoreactive local interneurons in the AL (Seki et al., 2010), thereby demonstrating specificity for GABAergic interneurons in the antennal lobe of Drosophila, similar to our analysis on the same type of neuron in the same species at the larval stage. Even Larkin et al. (2010), also using rabbit anti-GABA, showed a similar pattern of GABA-positive neurons within the larval antennal lobe in comparison with our study.

\section{Anti-CD2}

Anti-CD2 was raised in mouse and was shown to recognize the rat CD2 cell surface antigen, a 50-54-kDa glycoprotein expressed by thymocytes and mature $T$ cells (Whiteland et al., 1995). This antibody recognizes the ectopically expressed CD2 protein as demonstrated by the absence of labeling in wild-type brains (Bader et al., 2007). No labeling was seen in our preparations when mouse anti-rat CD2 was used as the primary antibody for the larval brains unless the GAL4 drove expression of CD2. More details on each antibody are given in Table 1.

\section{RESULTS}

\section{Expression patterns of 16 candidate interneuron-specific GAL4 lines in the larval antennal lobe}

To identify neurons in the larval AL, we crossed 16 candidate GAL4 lines (see Materials and Methods), many of which were reported to label local interneurons in the adult AL (Chou et al., 2010) to UAS-Cameleon 2.1 (Cam; Diegelmann et al., 2002), whose expression was studied by applying an antibody against GFP. Anti-Dlg staining was used to visualize the larval $\mathrm{AL}$ and other prominent landmarks as described before (Masuda-Nakagawa et al., 2005). In most of these lines, expression was seen in various regions of the central nervous system (CNS), such as central brain, optic lobes, mushroom bodies, tritocerebral/suboesophageal area (for simplicity called subesophageal ganglion [SOG] hereafter) or ventral nerve cord. 
TABLE 1.

Primary Antibodies Used

\begin{tabular}{|c|c|c|c|c|}
\hline Antibody & Animal & $\begin{array}{l}\text { Working } \\
\text { dilution }\end{array}$ & Immunogen & Source \\
\hline Anti-GFP & Rabbit & $1: 1,000$ & $\begin{array}{l}\text { Purified green fluorescent protein (GFP), a } 27-k \mathrm{Da} \text { protein } \\
\text { derived from the jellyfish Aequorea Victoria }\end{array}$ & $\begin{array}{l}\text { Molecular Probes, } \\
\text { A6455 }\end{array}$ \\
\hline Anti-GFP & Sheep & $1: 1,000$ & $\begin{array}{l}\text { Purified green fluorescent protein (GFP), a } 27-k \text { Da protein } \\
\text { derived from the jellyfish Aequorea Victoria }\end{array}$ & $\begin{array}{l}\text { Serotec, } \\
\quad 4745-1051\end{array}$ \\
\hline Anti-DLG & Mouse & $1: 20$ & $\begin{array}{l}\text { Smal-EcoRV fragment that includes the second PDZ domain } \\
\text { of DLG fused to GST (Amino acid range of the PDZ domain: } \\
\text { PGSRYASTNVLAAVPPGTPRAVSTEDITREPRTITIOKGPQGL } \\
\text { GFNIVGGEDGGIYVSFILAGGPADLGSELKRGDQLLSVNNVN } \\
\text { LTHATHEEAAQALKTSGGVVTLLAQYRPEEYNRFEARIOELKO } \\
\text { OAALGAGGSGTLLRTTOKRSLYVRALFDYDPNRDDGLPSRGLP FKHGDI) }\end{array}$ & 4F3, DSHB \\
\hline Anti-ChAT & Mouse & $1: 00$ & 80-kDa Drosophila ChAT protein & 4B1, DSHB \\
\hline Anti-CD8 & Rat & 1:00 & $\alpha$-Chain of mouse CD8 protein & Caltag, RM2200 \\
\hline Anti-GABA & Rabbit & 1:00 & GABA conjugated with BSA & Sigma, A2052 \\
\hline Anti-CD2 & Mouse & $1: 100$ & Activated rat T-helper cell homogenate & Serotec, MCA154R \\
\hline
\end{tabular}

H24, HB8-145, LCCH3, Nan-GAL4, and NP1227 did not label any intrinsic neurons of the larval $\mathrm{AL}$, but the remaining lines showed distinct expression in such neurons (Fig. 1). Three lines that were chosen for single-cell analysis, 189Y-GAL4; mb247-GAL80, NP2426-GAL4, and NP3056-GAL4 are described in detail below. NP1612, Krasavietz-GAL4, and OK 107 labeled variable numbers of neurons that densely innervated the entire AL. The cell bodies of most of these neurons were located ventrolateral of the AL, but in Krasavietz-GAL4 a few of them formed a dorsolateral cluster.

The neurons visualized by HB4-93 and NP0385 belonged to the same two clusters, with a relatively stronger contribution of dorsolateral neurons. In contrast to the former lines, the AL neurons labeled by these two lines established sparse innervation in the AL but massive arborizations in the SOG. C739, GH298, and NP6277 all showed expression in large numbers of cells situated around the lateral edge of the AL. Many of them established arborizations in the AL, but others were probably unrelated to the $\mathrm{AL}$.

\section{Interneurons in the larval antennal lobe revealed by $189 \mathrm{Y}-\mathrm{GAL} 4$; mb247-GAL80, NP2426-GAL4, and NP3056-GAL4: patterns, neurotransmitter identities, and larval development}

The neurons studied here displayed many of the anatomical properties of AL local interneurons. However, nearly all of them established extra arborizations in the SOG or even in higher brain centers. Moreover, because the border between the AL and the adjacent SOG is ill defined, a rigorous distinction between neurons restricted to the AL and those establishing additional arbors in the SOG was often not possible. Consequently, we chose the neutral term antennal lobe neurons (ALNs) in the following description.

Evidence from 15 ALs showed that the first of three lines studied in detail, 189Y-GAL4; mb247-GAL80; UASCam, labeled five to six ALNs (Fig. 2A). We used UASCam in our study as it provides two binding sites for the GFP antibody and therefore leads to an increased fluorescence (see also Selcho et al., 2009). The ALN cell bodies were located ventrolateral of the AL, either close to it (in one to two ALNs) or at some distance (for most ALNs). Arborizations were essentially restricted to the AL, although many preparations displayed a weak innervation in the adjacent SOG. Judged from the total pattern, the AL neuropil appeared to be homogeneously innervated (Fig. 2A).

The expression pattern of the second line, NP2426GAL4; UAS-Cam (11 ALs analyzed), included two clusters of strongly labeled ALNs, comprising a total of about six to eight neurons (Fig. 2B). Two to three of them had their cell bodies located ventrolateral of the AL (similar to most of the 189Y-ALNs), whereas four to five additional ALNs belonged to a dorsolateral cell cluster. The NP2426-GAL4 pattern revealed strong arborizations in both $\mathrm{AL}$ and SOG (Fig. 2B) and weaker branching in parts of the MBs (not shown). The third line, NP3056-GAL4 (six ALs studied), showed expression either in a single ventrolateral and a single dorsolateral ALN (Fig. 2C) or in two dorsolateral ALNs (not shown). Owing to the small number of labeled neurons, the density of innervation in the AL was inferior to those in the other two lines. Similar to NP2426-GAL4, additional strong arborizations occurred in the adjacent SOG (Fig. 2C).

We also tested the neurotransmitter identity of the ALNs revealed by 189Y-GAL4; mb247-GAL80 (Fig. 2D-G), 


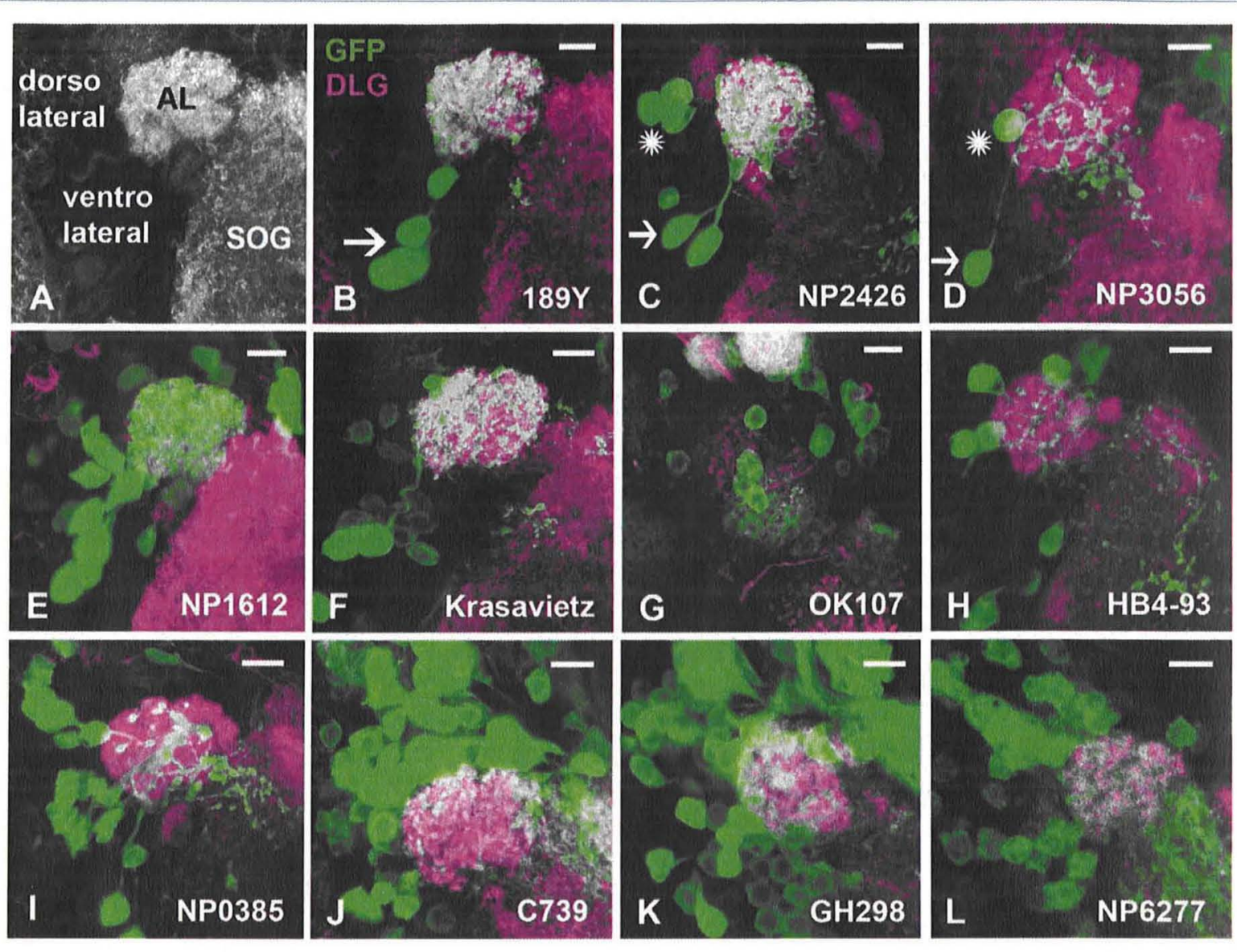

Figure 1. A-L: Expression patterns of $11 \mathrm{GAL} 4$ lines displaying neurons of the larval $\mathrm{AL}$. In lines whose expression pattern includes only a few neurons, a ventrolateral cluster of cell bodies (arrows) can be distinguished from a dorsolateral cluster (asterisks). For more details, see text. A: Orientation of the $\mathrm{AL}$ with respect to the SOG and the position of the two cell body clusters. Green, anti-green fluorescent protein (GFP) staining; magenta, anti-Discs Large (anti-DLG) staining. Panels represent Z-projections comprising 9-28 confocal planes. Scale bar $=10 \mu \mathrm{m}$ in $\mathrm{A}-\mathrm{L}$.

NP2426-GAL4 (Fig. 2H-K), and NP3056-GAL4 (Fig. 2LO). Compatible with a previous report using NP2426GAL4 (Asahina et al., 2009), anti-GABA immunocytochemistry revealed overlap with most of these neurons (Fig. 2F,J,N), except for rare dorsolateral neurons (Fig. $2 \mathrm{H}, \mathrm{I}, \mathrm{J})$. In contrast, in none of the three strains did any of the ALNs coincide with anti-ChAT staining (Fig. 2G,K,O).

Two clusters of ALNs comprising six to eight neurons in total, located ventrolateral and dorsolateral of the AL, were visualized by NP2426-GAL4 already in the first larval instar (four ALs analyzed) (Fig. 3A). Their numbers remained constant through the second larval instar ( $n=$ 4) (Fig. 3B), feeding third instar ( $n=5$ ) (Fig. 3C), and wandering third instar ( $n=9$ ) (Fig. 3D). However, the density of innervation of the $A L$ and the adjacent SOG appeared to increase during this period. Taken together, these data suggest that the NP2426-positive ALNs are of embryonic origin. However, whether the innervation patterns of indi- vidual neurons are related to their birthdates remains unknown.

\section{Types of interneurons innervating the larval antennal lobe}

To better characterize the ALNs, we generated flp-out clones in the three selected GAL4 lines. In general, most of the ALNs show a surprising degree of variability in their innervation patterns, making a transparent classification difficult. Therefore we have chosen a restrictive approach for the categorization of ALNs, which is based mainly on cell cluster origin, major differences in $A L$ innervation, and the presence or absence of SOG and MB innervation, criteria that are unambiguous. Differences in SOG innervation patterns were not taken into account due to their extreme variability. Our approach thus allows for a distinction of different ALN types and subtypes. The 


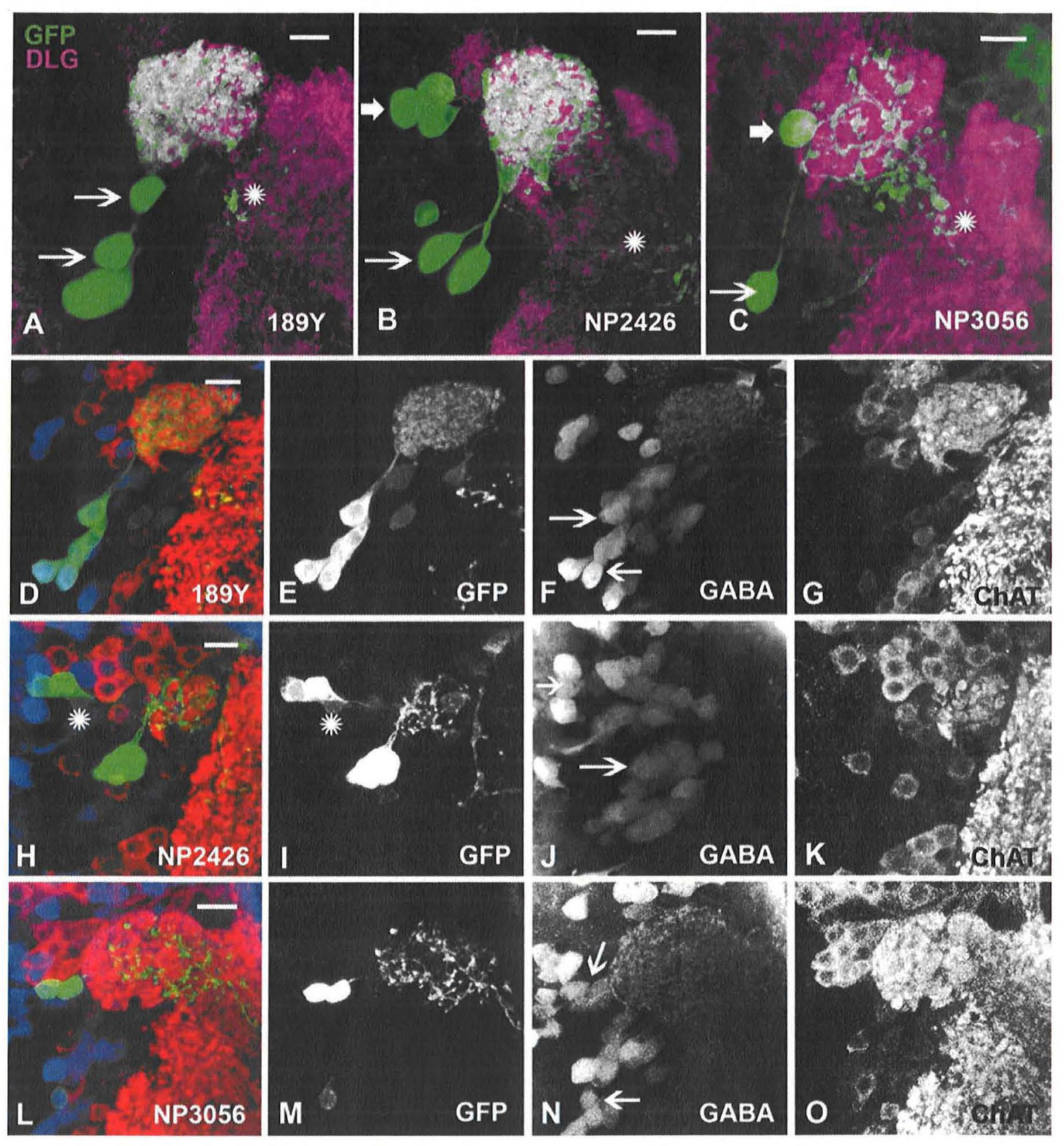

Figure 2. Patterns and neurotransmitter properties of neurons in the larval AL as revealed by three selected GAL4 lines. A: 189Y-GAL4; mb247-GAL80 labels AL neurons (ALNs) whose cell bodies are located ventrolateral of the AL (thin arrows). B: NP2426-GAL4 displays two clusters of ALNs, ventrolateral (thin arrow) and dorsolateral (bold arrow) of the AL. In both lines ALNs densely innervate the AL and send extra processes into the adjacent SOG (asterisks), especially in NP2426-GAL4. C: NP3056-GAL4 also displays ventrolateral ALNs (thin arrow) and dorsolateral ALNs (bold arrow). Innervation appears sparse in the AL but prominent in the adjacent SOG (asterisk). D,H,L: combined anti-GABA staining (blue) and anti-ChAT staining (red) in 189Y-GAL4; mb247-GAL80 (D), NP2426-GAL4 (E), and NP3056-GAL4 (F). E,I,M: GFP expression. F,J,N: Anti-GABA staining. G,K,O: Anti-ChAT staining. Anti-GABA immunocytochemistry overlaps with most of the neurons revealed by the three lines (F,J,N, arrows), except for rare dorsolateral neurons in NP2426-GAL4 (I,J; asterisks). In none of the strains do any of the ALNs coincide with anti-ChAT staining $(G, K, O)$. A-D,H,L: anti-GFP staining (green). A-C: Anti-DLG staining (magenta). Panels represent Z-projections comprising 10-32 confocal planes. In all panels, dorsal is on top and lateral to the left. Scale bar $=10 \mu \mathrm{m}$ in $A-C, D$ (applies to D-G), H (applies to $H-K$ ), and L (applies to $L-O$ ). 


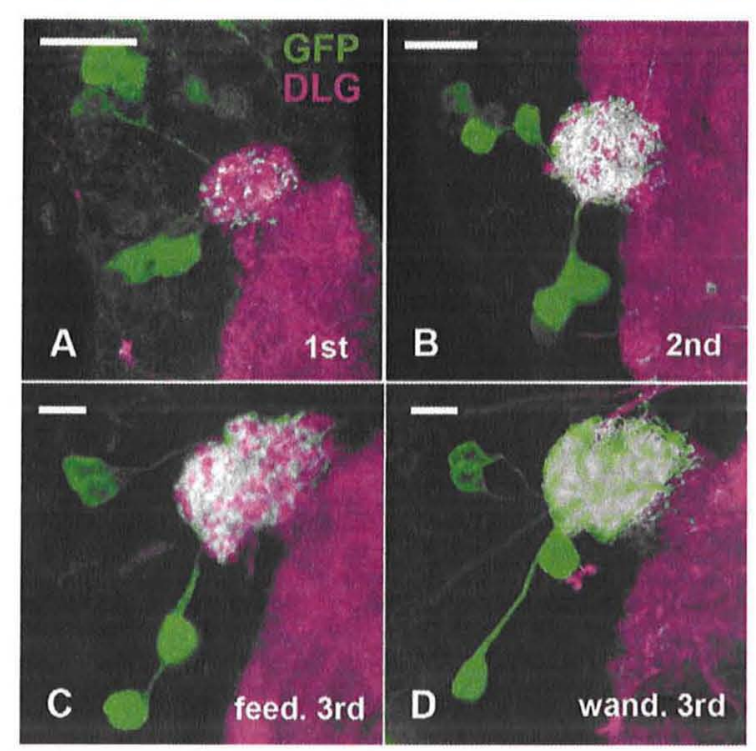

Figure 3. A-D: The two clusters of ALNs visualized by NP2426GAL4 remained constant from first larval instar (A) through second larval instar (B), and feeding and wandering third larval instar ( $C$ and $D$, respectively). Green, anti-GFP staining; magenta, anti-DLG staining. Panels represent Z-projections comprising 8-21 confocal planes. Dorsal is on top, lateral to the left. Scale bar $=10 \mu \mathrm{m}$ in A-D. subtypes we describe show considerable variation for certain pattern elements such as the innervation of distinct AL glomeruli. Additional subdivisions thus remain theoretically possible, but are unlikely given the restricted numbers of neurons in the larva. For a detailed overview, see also Table 2.

Neurons were identified in single-cell or two-cell clones. Two related but surprisingly variable neuronal morphologies collectively termed type 1 ALNs were revealed by clones of 189Y-GAL4 and NP2426-GAL4. From 36 ALNs studied in 189Y-GAL4; mb247-GAL80 and 40 ALNs analyzed in NP2426-GAL4 (Fig. 4), 26 neurons termed subtype $1 \mathrm{~A}$ ALNs established arborizations exclusively in the AL (Fig. 4A,B). The remaining 21 subtype $1 \mathrm{~B}$ ALNs showed an extra innervation in the adjacent SOG that was either weak $(n=13)$ or more prominent $(n=8)$ (Fig. 4C,D). Therefore type $1 \mathrm{ALNs}$ fall into subtypes A and $B$ based on the absence or presence of SOG innervation, respectively. The patterns of SOG arborization were very variable, with scattered arbors occasionally approaching the midline. The fact that many intensely stained ALNs did not display any trace of an SOG projection suggests that subtype $1 \mathrm{~A}$ morphology is real rather than being due to incomplete reporter expression. The large majority of ALNs innervated the AL homogeneously,

TABLE 2.

Summary of the Different Types of Antennal Lobe Neurons

\begin{tabular}{|c|c|c|c|c|c|}
\hline $\begin{array}{c}\text { Cell type } \\
\text { and subtype }\end{array}$ & AL innervation ${ }^{1}$ & Cell cluster ${ }^{1}$ & SOG and MB innervation ${ }^{2}$ & GAL4 line & $\begin{array}{c}\text { No. of } \\
\text { hits }\end{array}$ \\
\hline \multicolumn{6}{|l|}{ Type 1} \\
\hline Type 1A & $\begin{array}{l}\text { Homogenous innervation of the } \mathrm{AL} \text {, } \\
\text { sometimes less intense in the medial half }\end{array}$ & $\begin{array}{l}\text { Ventrolateral } \\
\text { cluster }\end{array}$ & No & $\begin{array}{l}\text { 189y GAL4, } \\
\text { NP2426 }\end{array}$ & 26 \\
\hline Type 1B & $\begin{array}{l}\text { Homogenous innervation of the AL, } \\
\text { sometimes less intense in the medial half }\end{array}$ & $\begin{array}{l}\text { Ventrolateral } \\
\text { cluster }\end{array}$ & $\begin{array}{l}\text { Variable SOG innervation } \\
\text { ranging from weak } \\
\text { to widespread }\end{array}$ & $\begin{array}{l}189 y \text { GAL4, } \\
\text { NP2426 }\end{array}$ & 21 \\
\hline \multicolumn{6}{|l|}{ Type 2} \\
\hline Type 2A & $\begin{array}{l}\text { Sparse AL innervation restricted to } \\
\text { interglomerular spaces }\end{array}$ & $\begin{array}{l}\text { Ventrolateral } \\
\text { cluster }\end{array}$ & Massive SOG innervation & NP2426 & 8 \\
\hline Type 2B & $\begin{array}{l}\text { Sparse AL innervation restricted to } \\
\text { interglomerular spaces of a small medial area }\end{array}$ & $\begin{array}{l}\text { Ventrolateral } \\
\text { cluster }\end{array}$ & Massive SOG innervation & NP3056 & 3 \\
\hline Type 3 & Dense innervation of the lateral AL half & $\begin{array}{l}\text { Dorsolateral } \\
\text { cluster }\end{array}$ & Few extra arbors in SOG & NP2426 & 4 \\
\hline Type 4 & Sparse innervation of the posterior $A L$ half & $\begin{array}{l}\text { Dorsolateral } \\
\text { cluster }\end{array}$ & Massive SOG innervation & $\begin{array}{l}\text { NP2426, } \\
\text { NP } 3056\end{array}$ & 7 \\
\hline \multicolumn{6}{|l|}{ Type 5} \\
\hline Type 5A & $\begin{array}{l}\text { Dense and homogeneous innervation } \\
\text { of the ventromedial half }\end{array}$ & $\begin{array}{l}\text { Dorsolateral } \\
\text { cluster }\end{array}$ & Massive SOG innervation & NP2426 & 14 \\
\hline Type 5B & $\begin{array}{l}\text { Dense and homogeneous innervation } \\
\text { of the ventromedial half }\end{array}$ & $\begin{array}{l}\text { Dorsolateral } \\
\text { cluster }\end{array}$ & $\begin{array}{l}\text { Massive SOG and MB } \\
\text { lobe innervation }\end{array}$ & NP2426 & 3 \\
\hline Type $5 C^{3}$ & $\begin{array}{l}\text { Dense and homogeneous innervation } \\
\text { of the ventromedial half }\end{array}$ & $\begin{array}{l}\text { Dorsolateral } \\
\text { cluster }\end{array}$ & $\begin{array}{l}\text { Massive SOG and MB } \\
\text { lateral appendix } \\
\text { innervation }\end{array}$ & NP2426 & 1 \\
\hline
\end{tabular}

Abbreviations: $\mathrm{AL}$, antennal lobe; SOG, suboesophageal ganglion; $\mathrm{MB}$, mushroom body.

'AL innervation and cell cluster origin were used as criteria to distinguish different cell types.

${ }^{2}$ SOG innervation patterns do not allow for a classification due to variability. Classification was only possible for type 1 , based on the absence or presence of SOG innervation in subtypes $1 \mathrm{~A}$ and $1 \mathrm{~B}$.

${ }^{3} \mathrm{As}$ we found only one clone for this subtype, it may also belong to type $5 \mathrm{~B}$. 


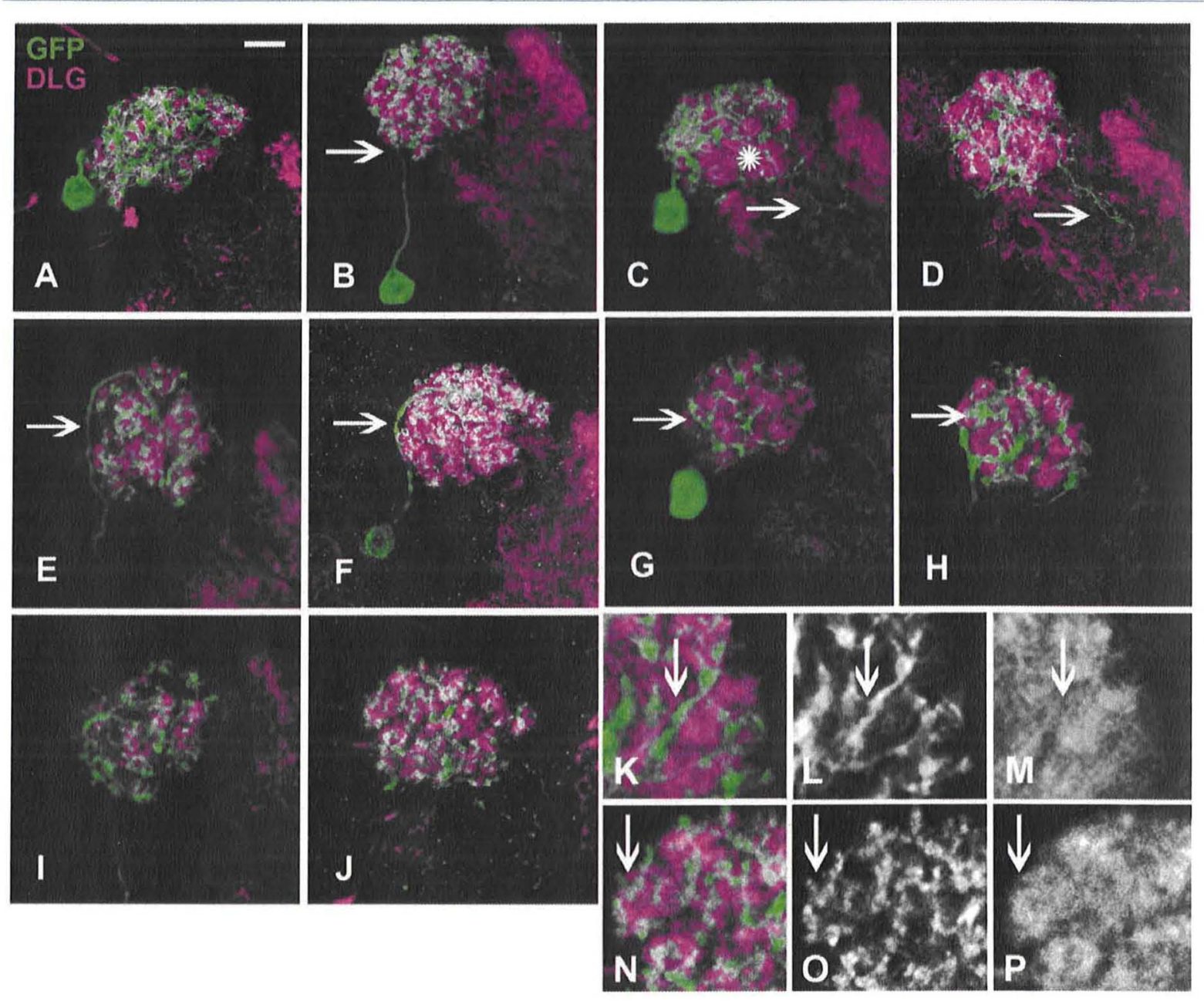

Figure 4. Cellular anatomy of type 1 ALNs displayed in flp-out clones of 189Y-GAL4; mb247GAL80, and NP2426-GAL4. A-D: Subtype 1A ALNs restrict their arbors to the AL. (A,B), whereas subtype $1 B$ ALNs establish an extra innervation in the adjacent SOG (C,D; arrows). In general, the AL is homogeneously innervated (A-D), but, exceptionally, branching is less dense in its medial half (C; asterisk). The cell body fiber of both subtypes either enters the AL straight after reaching the ventrolateral corner of the AL (B; arrow) or loops along its lateral edge before branching (E,F; arrows). Secondary processes pass essentially via interglomerular spaces (G,H; arrows) before sending tightly branched tertiary processes into the glomeruli proper (I,J). E-J: Different single-cell clones. K-M: Higher magnification of secondary processes (arrows) in the interglomerular spaces. L,M: Only the anti-GFP or anti-DLG staining, respectively. N-P: Higher magnification of tertiary processes innervating AL glomeruli (arrows). O,P: Only the anti-GFP or anti-DLG staining, respectively. Green, anti-GFP staining; magenta, anti-DLG staining. Panels represent Z-projections comprising 3-10 confocal planes (single planes in G,H). Dorsal is on top and lateral to the left. Scale bar $=10 \mu \mathrm{m}$ in $\mathrm{A}$ (applies to A-J; K-M and N-P show higher magnifications of $\mathrm{H}$ and J, respectively).

ostensibly covering all of its glomeruli with similar density. Exceptionally, branching tended to be less intense in the medial half of the AL (Fig. $4 \mathrm{C}$ ) or some glomeruli received slightly stronger innervation (not shown), but whether these patterns were genuine or due to unequal reporter expression remains an open question.

For 31 type 1 ALNs, the primary process extending from the cell body split up into secondary processes immediately upon reaching the AL neuropil at the ventrolateral edge (Fig. 4B). In the remaining 16 cases, the primary process extended laterally around the AL neuropil before establishing secondary processes in the dorsolateral quadrant of the AL (Fig. 4E). The secondary processes passed essentially via interglomerular spaces (Fig. $4 \mathrm{G}, \mathrm{H}, \mathrm{K}-\mathrm{M}$ ) before sending tightly branched tertiary processes into the glomeruli proper (Fig. 4I,J,N-P). The cell bodies of 12 type $1 \mathrm{ALNs}$ were located close to the $\mathrm{AL}$ neuropil (Fig. 4A), and those of the remaining 35 type 1 ALNs were at some distance (Fig. 4B). These proportions reflect the cell body positions known from the total pattern of 189Y-GAL4 (cf. Fig. 2A). The position was not correlated with either the trajectory of the primary process 
or with the presence or absence of an extra SOG innervation.

Hence, subtype $1 \mathrm{~A}$ and $1 \mathrm{~B}$ ALNs share cell body position and arborization patterns in the $\mathrm{AL}$, but differ, respectively, in the absence or presence of an extra innervation in the SOG. The lack of correlation between cell body position and SOG innervation and the variable arborization patterns in the SOG suggest that the establishment and the extent of this extra innervation may not be strictly programmed.

The 40 ALNs analyzed in NP2426-GAL4 clones and an additional 10 single-cell clones in NP3056-GAL4 revealed another seven anatomically distinct subtypes of ALNs. Type 2 ALNs $(n=11)$, which also had their cell body in the ventrolateral cluster, were characteristic for sparse branching in the $A L$ but massive innervation of the SOG. Subtype 2A ALNs $(n=8)$ (Fig. $5 A-D)$ displayed a small number of stout, prominent processes in the $A L$ that extended via interglomerular spaces (Fig. 5A,B). Despite robust reporter expression, discrete side branches into the glomeruli proper were not observed. We also note that these ALNs innervated mostly the posterior half of the AL (Fig. 5D). In subtype 2B ALNs ( $n=3)$, the primary process extended straight through the AL neuropil and established arborizations exclusively in a small medial area of the AL adjacent to the SOG (Fig. 5E,F). Both subtype $2 \mathrm{~A}$ and $2 \mathrm{~B}$ ALNs were characteristic for massive, highly variable arbors in broad areas of the SOG, up to the midline (Fig. 5A-F).

In contrast to type 1 and 2 ALNs, the cell bodies of type $3 \mathrm{ALNs}(\mathrm{n}=4)$ belonged to the dorsolateral cluster (Fig. 6A,B). These neurons densely innervated the lateral half of the AL (largely bypassing the medial half) (Fig. $6 \mathrm{~B}, \mathrm{C})$ and established a few extra arbors in the adjacent SOG (Fig. 6A,B).

Type 4 ALNs also had a dorsolateral cell body (Fig. $6 \mathrm{D}, \mathrm{E})$, but, similar to subtype $2 \mathrm{~A}$ ALNs, branching in the $A L$ was sparse (Fig. 6E) but stronger in its posterior half (Fig. 6F), and the innervation of the SOG was massive (Fig. 6D,F)

Type 5 ALNs $(n=17)$ showed three major characteristics. First, their cell bodies belonged to the dorsolateral cluster (Fig. 7A-D,F,G,IJ). Second, their primary process was thicker than in other ALN classes; it generally entered the AL neuropil at an anteroventral position and often split initially into two thick secondary processes (Fig. 7A-C). Third, arborizations covered mostly the ventromedial half of the AL neuropil, leaving the dorsolateral half empty (Fig. 7A-G,J). Given that this pattern was strong and similar in all clones, the lack of innervation of the dorsolateral half is unlikely to be an artifact. In four cases, a particular glomerulus in the posterior half, called 1a (Masuda-Nakagawa et al., 2009), appeared more
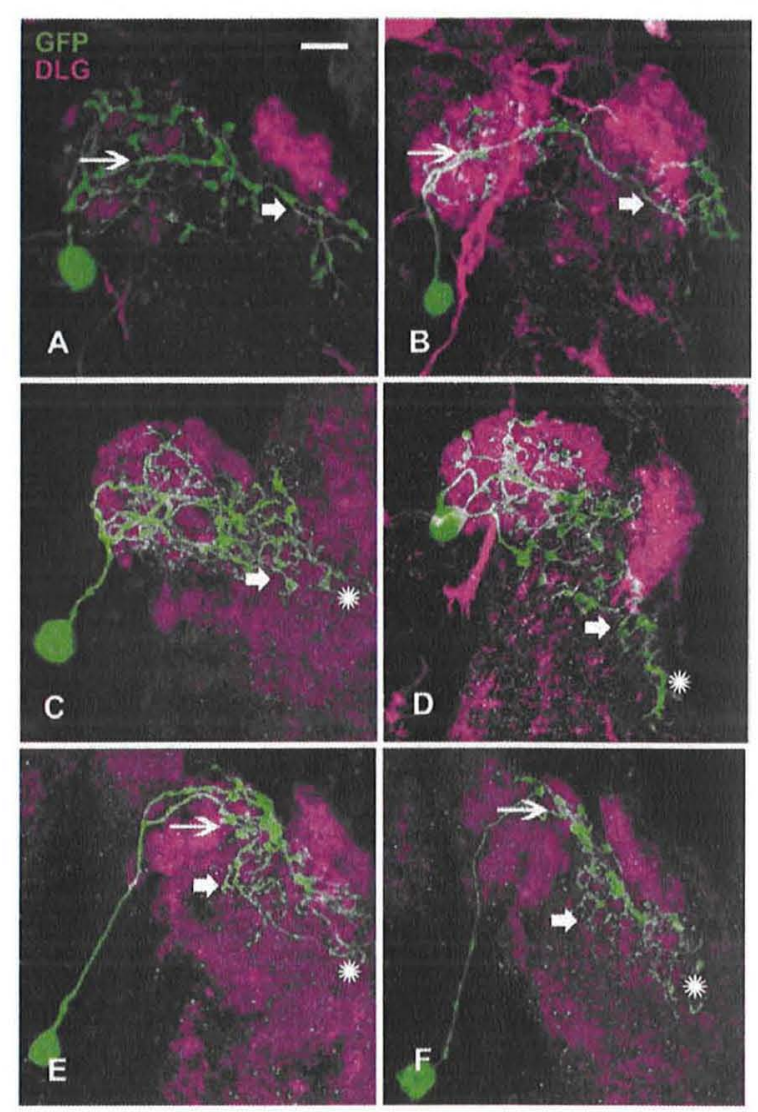

Figure 5. Type 2 ALNs revealed in flp-out clones of NP2426GAL4 (A-D) and NP3056-GAL4 (E,F) belong to the ventrolateral cluster. A-D: Subtype 2A ALNs innervate the AL via a few prominent secondary processes ( $A, B$; thin arrows), which sparsely branch in glomeruli. The anterior half of the $A L(A, B)$ is often less densely covered than the posterior half $(C, D)$. E,F: Subtype 2B $A L N s$ innervate exclusively a small medial area of the $A L$ adjacent to the SOG (thin arrows). Both subtype $2 \mathrm{~A}$ and $2 \mathrm{~B}$ ALNs establish massive, variable arborization patterns in the SOG (A-F; bold arrows) frequently up to the midline (C-F; asterisks). Green, antiGFP staining; magenta, anti-DLG staining. Panels represent Z-projections comprising 6-29 confocal planes. Dorsal is on top and lateral to the left. Scale bar $=10 \mu \mathrm{m}$ in A (applies to A-F).

densely innervated than other glomeruli (Fig. 7E). In contrast to class $2 \mathrm{ALNs}$, the AL innervation of type $5 \mathrm{ALNs}$ was dense and homogeneous, with secondary processes extending largely via interglomerular spaces and tertiary processes terminating in the glomeruli. However, similar to class 2 ALNs, parallel processes passed into the SOG and established prominent arborizations over a wide but variable area up to the midline and sometimes even beyond (Fig. 7C-F,H).

Type 5 ALNs fall into three subtypes: subtype 5 A ALNs $(n=14)$ are characterized exclusively by the features mentioned above. Subtype 5B ALNs $(n=3)$ displayed the 

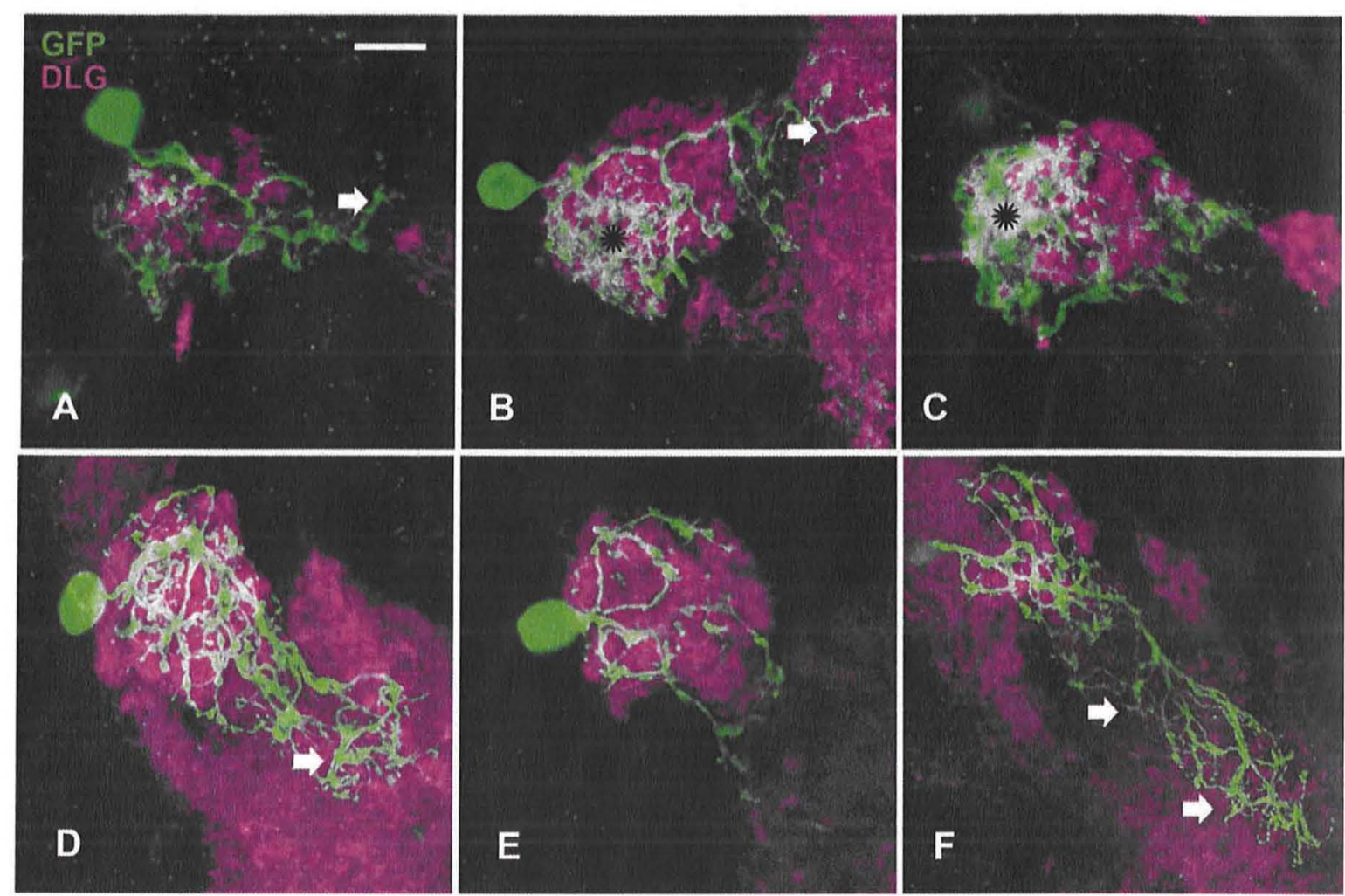

Figure 6. Type 3 ALNs (A-C) and type 4 ALNs (D-F). A-C: Type 3 ALNs shown in flp-out clones of NP2426-GAL4 are part of the dorsolateral cluster. They densely innervate the lateral half of the AL (B,C; asterisks) and establish extra arbors in the adjacent SOG (A,B; bold arrows). D-F: Type 4 ALNs also have a dorsolateral cell body (D,E), but branching in the AL is sparse and stronger in its posterior half (cf. $\mathrm{E}$ and $\mathrm{F}$ ) and innervation of the SOG is massive (D,F; bold arrows). E,F: Different focal planes of the same single-cell clone. Green, antiGFP staining; magenta, anti-DLG staining. Panels represent Z-projections comprising 4-23 confocal planes. Dorsal is on top and lateral to the left. Scale bar $=10 \mu \mathrm{m}$ in A (applies to A-F).

same patterns in the AL and SOG (Fig. 7G-I) but sent an axon via a pathway extending lateral of the antennocerebral tract toward a prominent subregion of the mushroom bodies called the lateral appendix (Pauls et al., 2010b) (Fig. 7l). This axon established terminal arborizations medial to the lateral appendix and then split into two branches that formed additional terminals around the base of the mushroom body vertical lobe (Fig. 7l). A related type of neuron $(n=1)$, termed subtype $5 \mathrm{C}$ ALN (Fig. 7J,K), was almost identical to 5B ALNs, except for the axon extending into the mushroom body area. Instead of staying outside the lateral appendix, this process established terminals right inside this structure (Fig. 7K). In addition, the two terminal branches did not end at the base of the vertical lobe but climbed to its top before establishing terminals (not shown). However, it remains possible that $5 \mathrm{~B}$ ALNs represent an incompletely expressed version of $5 \mathrm{C}$ ALNs. Nevertheless, subtype $5 \mathrm{C}$ ALNs are intriguing because they interconnect primary olfactory and gustatory target regions with the mushroom bodies, three major areas implicated in chemosensory learning.

\section{Putative direction of signal transfer in antennal lobe neurons}

To elucidate likely input/output relations of ALNs in the $\mathrm{AL}$ and SOG, we crossed UAS-Dscam17.1-GFP, described as a putative postsynaptic marker (Wang et al., 2004a; Selcho et al., 2009) and UAS-nsyb-GFP, an established presynaptic marker (Ito et al., 1998; Selcho et al., 2009), with the three selected ALN GAL4 drivers. Each of these strains exhibited both massive Dscam 17.1 expression (Fig. 8A-C) and nsyb expression (Fig. 8D-F) in the AL. Regarding SOG processes, Dscam 17.1 expression was obvious in 189Y-GAL4; mb247-GAL80 (Fig. 8A) and in NP3056-GAL4 (Fig. 8C). Despite strong Dscam expression of NP2426-GAL4 in the AL, there were only weak traces of such label in the SOG (Fig. 8B). In contrast, in all three lines, nsyb expression was evident in the SOG, in a 

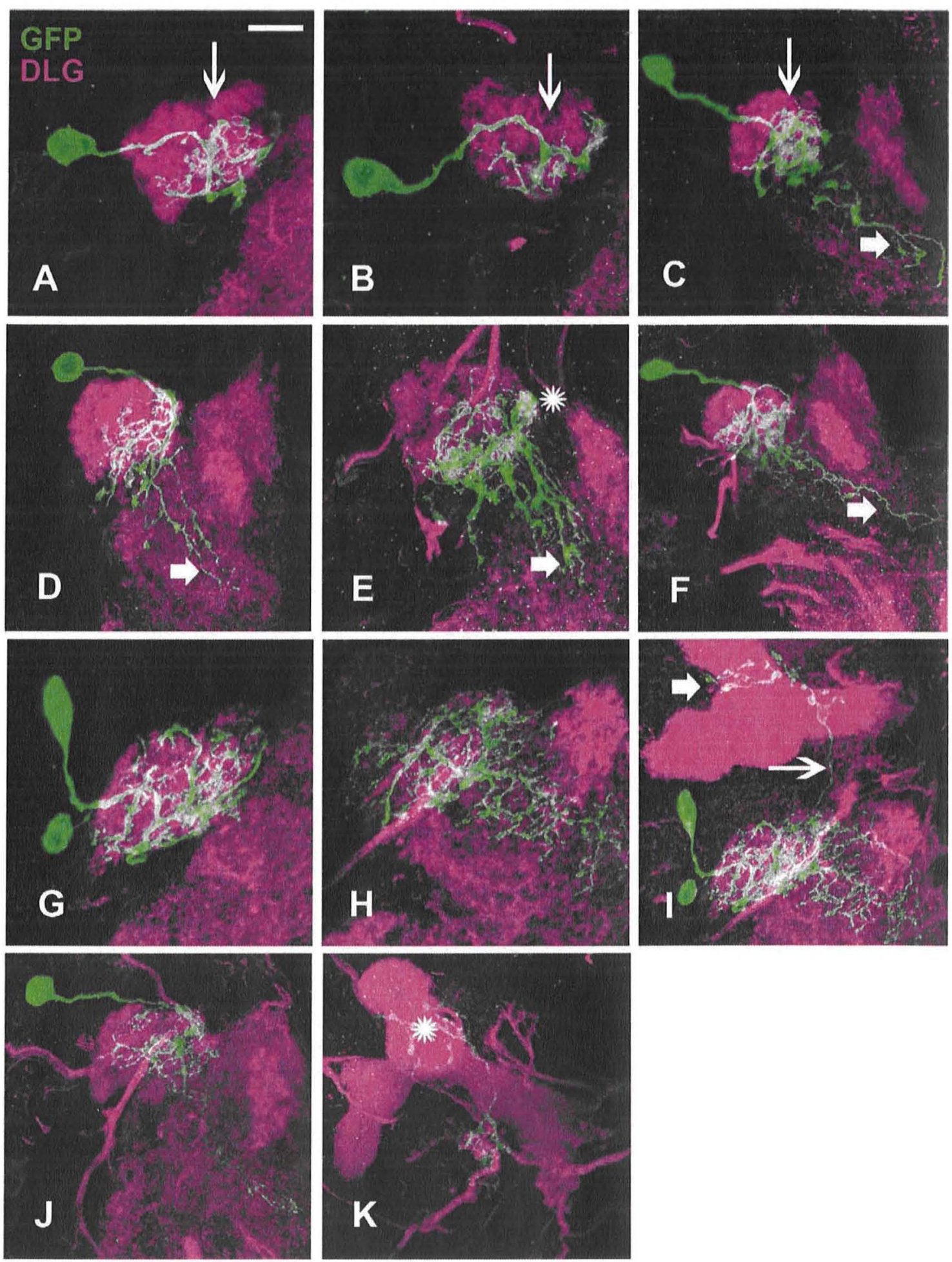

Figure 7. Type 5 ALNs displayed by flp-out clones of NP2426-GAL4 belong to the dorsolateral cluster (A-D,F,G,I,J). A-F: Subtype 5A ALNs have a thick primary process that enters the $\mathrm{AL}$ at an anteroventral position and often initially splits into two secondary processes (A-C; thin arrows). Arborizations cover exclusively the ventromedial half of the AL (A-F). Occasionally, glomerulus Or 1a receives a particularly dense innervation (E; asterisk). Parallel processes pass from the AL into the SOG and establish prominent but variable arborizations up to the midline (C-F; bold arrows). G-I: Subtype 5B ALNs establish arbors in the AL and SOG (H,I), similar to 5A ALNs, but in addition send an axon toward the lateral appendix of the MBs (I; thin arrow). Its terminals are located medial to the lateral appendix and around the base of the MB vertical lobe (I; bold arrow). J,K: Subtype 5C ALNs are almost identical to subtype 5B, except that the axon extending into the MB area forms terminals inside the lateral appendix (K; asterisk). Green, anti-GFP staining; magenta, anti-DLG staining. Panels represent Z-projections comprising 5-40 confocal planes. Dorsal is on top and lateral to the left. Scale bar $=10 \mu \mathrm{m}$ in A (applies to A-K). 


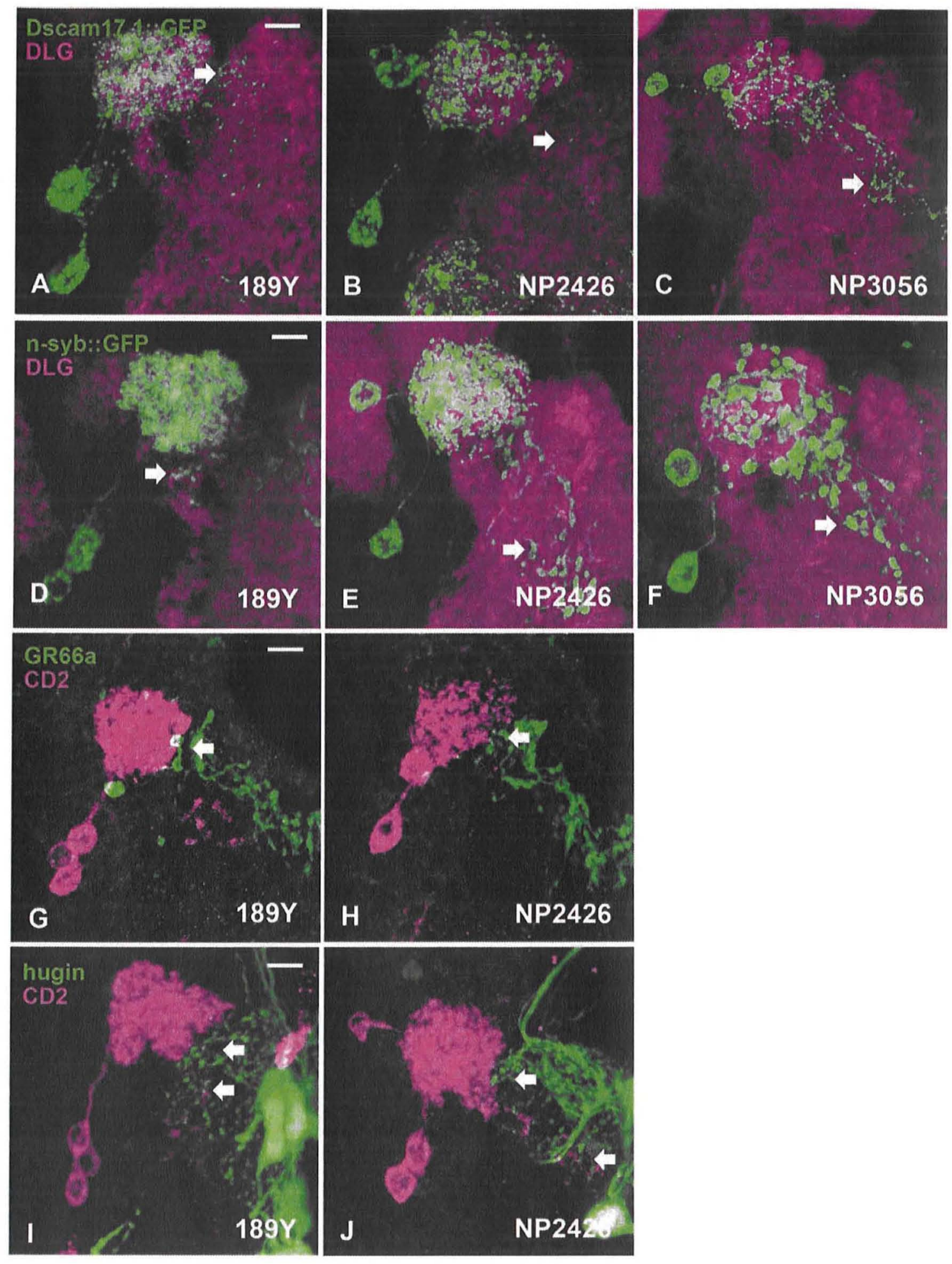

Figure 8. Likely synaptic input and output relations of $A L N$ s in the $A L$ and SOG (A-F) and overlap with Gr66a gustatory terminals (G,H) and hugin neurons (I,J) in the SOG. A,D,G,I: 189Y-GAL4; mb247-GAL80. B,E,H,J: NP2426-GAL4. C,F: NP3056-GAL4. Demonstration of putative postsynaptic sites by UAS-Dscam 17.1::GFP expression (A-C; green) and of putative presynaptic sites by UAS-nsyb::GFP expression (D-F; green). Arrows in A-J refer to expression in the SOG. G,H: Terminals of ALNs (magenta) and gustatory receptor neurons (green) in close proximity within the SOG (arrows). I,J: Processes of ALNs (magenta) and hugin neurons (green) in close neighborhood within the SOG (arrows). Panels represent Z-projections comprising 5-30 confocal planes. Magenta in A-F, anti-DLG staining. Dorsal is on top and ral to the left. Scale bar $=10 \mu \mathrm{m}$ in A (applies to A-C), D (applies to D-F), G (applies to G,H), and I (applies to I,J). 
pattern compatible with the anatomy of the ALN projections revealed by each of them (Fig. 8D-F). In conclusion, we suggest that the ALNs labeled by the three lines exhibit both input and output sites in the $A L$ and in their SOG projections, except for NP2426 ALNs, which appear to have less input in the SOG.

We then analyzed possible overlap of ALN processes in the SOG with the terminals of gustatory neurons responding to bitter taste, as visualized by Gr66a::GFP (Thorne et al., 2004; Wang et al., 2004b). As Gr66a is tagged by GFP, we drove UAS-CD2 by the different GAL4 lines for analyzing the GAL4 expression pattern (the same is also true in the following when using hugin::YFP). Although CD2 expression of the 189Y-GAL4; mb247-GAL80 and NP2426 driver lines was weaker than its Cam expression (Fig. 2A-C), ALN processes in the SOG were seen in close neighborhood to terminals of gustatory receptor neurons (Fig. 8G,H). Finally, we checked for overlap of ALN processes with neurons expressing the Hugin neuropeptide, cells that play crucial roles in feeding behavior (Melcher and Pankratz, 2005). As shown in Figure 81,J, hugin neurons and ALNs of the two driver lines have terminal processes located in close proximity. Taken together, the data indicate that larval ALNs may integrate olfactory cues with gustatory information as they are potentially associated with gustatory neurons and a neuronal circuit related to feeding. Our data are restricted to the two driver lines 189Y-GAL4; mb247-GAL80 and NP2426, as NP3056 only weakly expresses in two ALNs and was not reliably detected by the anti-CD2. Such a detection level problem was not seen when anti-GFP was used.

\section{Types of projection neurons innnervating the larval antennal lobe}

The different subtypes of uniglomerular PNs and their connectivity with individual calyx glomeruli have been analyzed in detail (Masuda-Nakagawa et al., 2009). The tool used for this study was the PN line GH146-GAL4 (Stocker et al., 1997; Heimbeck et al., 2001), which covers all AL glomeruli except 67b and 74a (Masuda-Nakagawa et al., 2009). As shown by four independent flp-out preparations, the expression pattern of NP2426-GAL4 included a PN that specifically innervated glomerulus $67 \mathrm{~b}$ (data not shown). The cell body of this neuron was located at the ventrolateral edge of the AL (data not shown), and its neurite extended through the inner antennocereberal tract (iACT; data not shown). Similar to other uniglomerular PNs, its dendritic arborizations covered a slightly larger area than the terminals of the corresponding olfactory receptor neuron $67 \mathrm{~b}$. As suggested by two flp-outs, the output area of the $67 \mathrm{~b} P N$ in the MB calyx corresponds to one of the vacant calyx glomeruli of the GH146-based
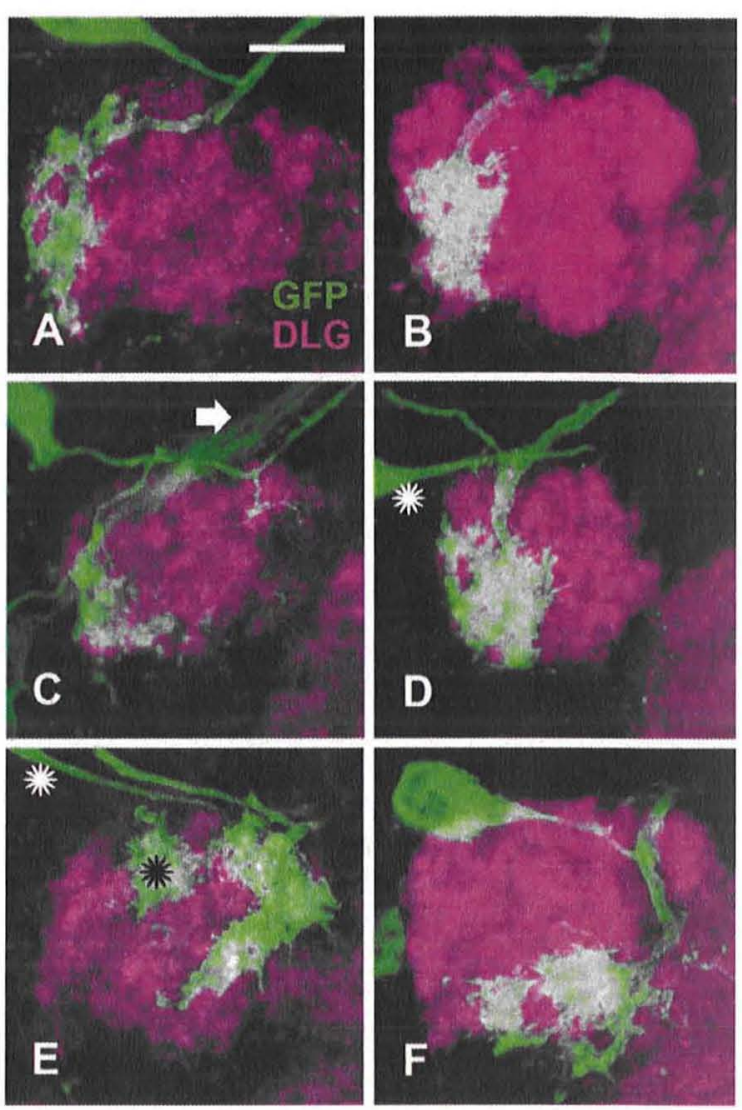

Figure 9. Wide-field PNs revealed by flp-out clones of $\mathrm{GH} 146$ GAL4. The areas of the AL covered by the dendritic arborizations largely exceed the size of individual glomeruli. A,B,F: Single-cell flp-outs. D,E: A second, uniglomerular PN (white asterisks) whose arborization region (black asterisk) is distinct from the wide-field PN. C: Includes the axons of immature, adult-specific PNs (bold arrow) that do not innervate the larval AL. Green, anti-GFP staining; magenta, anti-DLG staining. Panels represent Z-projections comprising 6-18 confocal planes. Dorsal is on top and lateral to the left. Scale bar $=10 \mu \mathrm{m}$ in A (applies to A-F).

map (Masuda-Nakagawa et al., 2009), termed A2 (Masuda-Nakagawa et al., 2010) (data not shown). Comparable to GH146 PNs, an extra process of the $67 \mathrm{~b}$ PN extended from the calyx into the lateral horn to establish a second terminal field (data not shown). A PN comprising spatially restricted dendritic arbors in the lateral area of the $\mathrm{AL}$ and an axon extending through the $\mathrm{iACT}$ was visible already in the first instar larva (data now shown).

At low frequencies $(n=15)$, flp-out clones in GH146GAL4 revealed the presence of PNs whose dendritic arbors occupied areas that visibly exceeded the size of individual glomeruli (Fig. 9A-F). They were often found in the lateral half of the AL (Fig. 9A-D). For example, the PN shown in Figure 9A covered at least glomeruli 30a, 45b, $63 \mathrm{a}$, and $94 \mathrm{~b}$. The precise areas filled by these wide-field 
PNs were extremely variable, i.e., none of the dendritic patterns of the 15 neurons identified were in fact identical. Moreover, none of the dendritic arbors appeared to respect glomerular borders. Given that GH146-GAL4 labels about 22 PNs, 19 of which are of the uniglomerular type (Masuda-Nakagawa et al., 2009), only three extra types are left. The fact that many more than three patterns occurred suggests that wide-field PNs differ across larvae.

\section{DISCUSSION}

ORNs and the majority of PNs restrict their arbors in the $\mathrm{AL}$ to single glomeruli and thus establish the conspicuous "vertical" circuitry of the olfactory pathway. Based on this straightforward design, much information has been collected in the past few years about the connectivity and functional role of these two cell types, in both adult and larval Drosophila. Local interneurons of the AL, which provide "horizontal" connections, were also known for a long time, although their role in odor processing was initially underestimated ( $\mathrm{Ng}$ et al., 2002; Wang et al., 2003). However, recent electrophysiological studies provided evidence of significant integration of odor information by local interneurons in the adult AL (Wilson et al., 2004; Wilson and Laurent, 2005; Bhandawat et al., 2007; Olsen et al., 2007; Olsen and Wilson, 2008). However, given the presence of inhibitory and excitatory cell types and of a wide variety of anatomical types (Das et al., 2008; Chou et al., 2010), a generalized concept of the functional role of local interneurons is still hard to obtain. Here we focus on the larval olfactory circuit, which shares the design of the adult circuit but includes 10-100 times fewer neurons (Masuda-Nakagawa et al., 2005, 2009; Ramaekers et al., 2005). We provide evidence that the horizontal connectivity in the larval AL differs largely from its adult counterpart, unlike the vertical circuitry, which is conserved between the adult and larval olfactory pathway.

\section{Cell types and wiring variability in the larval antennal lobe}

Surprisingly, only one of the nine anatomical subtypes of ALNs we describe ( $1 \mathrm{~A})$ is restricted to the AL neuropil and hence meets the definition of an olfactory local interneuron. In contrast, the eight remaining subtypes branch-in addition to the $\mathrm{AL}$-in the SOG, either adjacent to the $\mathrm{AL}$ or in a larger area up to the midline or even beyond. The assignment of ALNs to one of the five classes is straightforward, based on cell body position, morphology of the primary processes, density, and pattern of arborization in the $\mathrm{AL}$ and/or SOG and/or MB. Apart from these characteristics, we also observe vari- ability at the finer level of arborizations, which could reflect either incomplete reporter expression or true variability within cellular subtypes or even across larvae. Furthermore, the low number of identified clones for some subtypes of ALNs (subtype 2B, type 3, subtypes $5 B$ and $5 \mathrm{C})$ might limit our approach. Supporting the variability argument are the patterns of type $2 \mathrm{ALNs}$ in the AL and of type 2, 4, and 5 ALNs in the SOG.

In both the AL and SOG, the numbers and trajectories of major processes differ considerably. For example, type 5 ALNs may send either a single, profusely branching process from the AL to the SOG or up to a dozen parallel, relatively unbranched processes. These observations are hard to explain as an artifact. A good argument in favor of variability is provided by the fact that the different ALN morphologies observed, especially in the SOG, largely exceed the numbers of neurons labeled by the GAL4 drivers used. However, the possibility should not be excluded that the larval AL consists of ALNs to a greater extent than suggested by our approach, and therefore variation might be overestimated.

Even stronger evidence of wiring variability is demonstrated by the dendritic arborizations of wide-field PNs visualized in rare flp-outs of GH146-GAL4. This is quite remarkable because GH146-GAL4 labels about 22 PNs, 19 of which are of the uniglomerular type (Masuda-Nakagawa et al., 2009). This leaves only three extra types of PNs, i.e., much less than the observed wide-field patterns. Hence, we hypothesize that the morphology of wide-field PNs varies across larvae (Fig. 10B). However, we are not able to predict how completely the given data support this variation.

\section{Origin of larval antennal lobe neurons}

Our data suggest that ALNs are of embryonic rather than larval origin (Fig. 3). Whereas the developmental mechanisms that give rise to ORN and PN circuitry have been studied in great detail in Drosophila, only a few studies refer to the development of LNs or ALNs (Komiyama and Luo 2006; Jefferis and Hummel 2006; Rodriguez and Hummel, 2008; Das et al., 2008). For instance, intrinsic transcription factors as well as gradients of axon guidance molecules have been shown to control adult PN targeting, which is prespecified by lineage identity (deriving from an anterodorsal, a lateral, and a ventral neuroblast) and birth order (Jefferis et al., 2001; Komiyama et al., 2003; Spletter et al., 2007). Adult LNs arise throughout the proliferative cycle of the lateral neuroblast that begins in the embryo, whereas uniglomerular projection neurons arise later during the second larval instar (Das et al., 2008). Moreover, the lateral neuroblast requires the function of the cephalic gap gene empty spiracles for the development of olfactory interneurons (Das et al., 2008). 


\section{A}

\section{hardwired olfactory pathway}

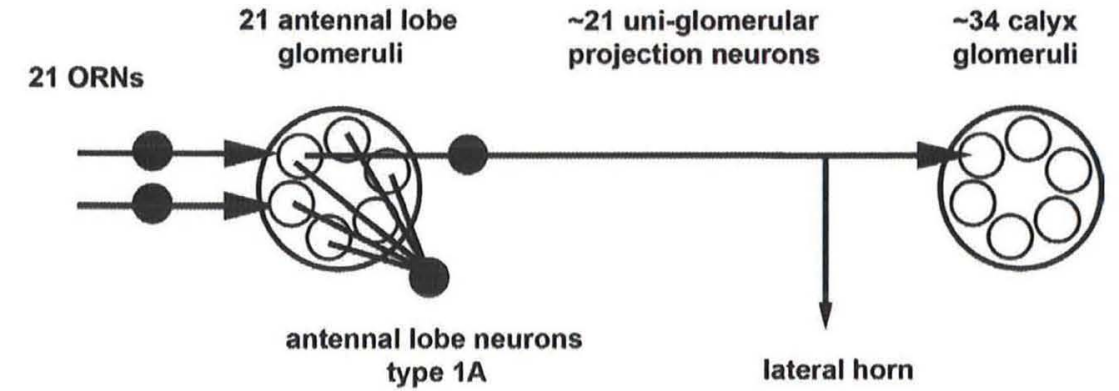

B

\section{individual variability within the olfactory pathway}

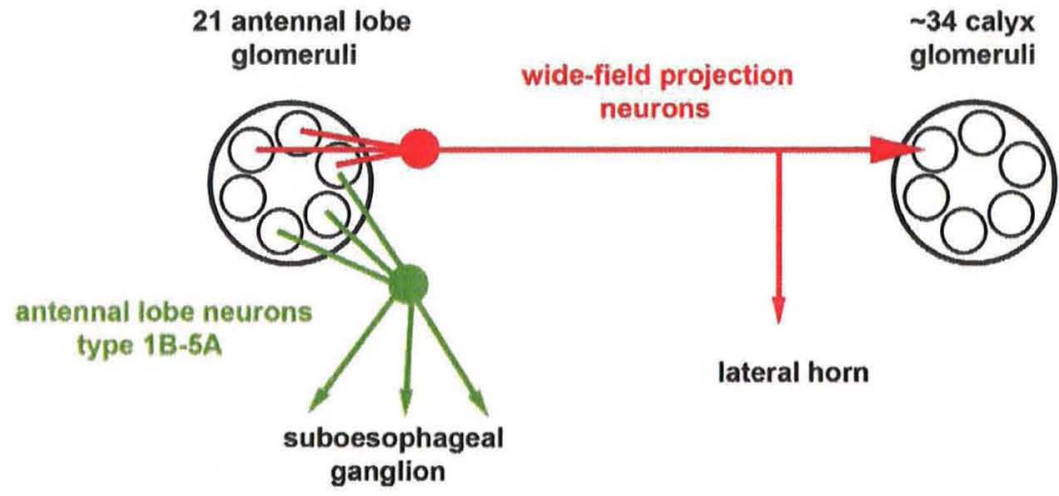

Figure 10. The larval olfactory system consists of hardwired components that do not differ between individuals. A: Olfactory receptor neurons, type $1 \mathrm{~A}$ antennal lobe neurons, and uniglomerular projection neurons. B: In contrast, wide-field projection neurons and type 1B-5A antennal lobe neurons vary between different larvae. Therefore wiring variability may be another principle of insect brain organization, in parallel with stereotypy.

Based on our description, it will now be possible to study the birth order and molecular factors underlying ALN development in detail.

\section{Variability as a principle of insect brain organization}

A number of recent reports indeed challenge the classical view that insect brain circuits are entirely or at least predominantly stereotyped. Interestingly, whereas the terminal arborization patterns of PNs in the lateral horn of adult Drosophila are quite stereotyped, the connectivity of the same neurons in the MB calyx shows a considerable degree of topographic and functional variability (Marin et al., 2002; WoNg et al., 2002; Jefferis et al., 2007; Murthy et al., 2008). More importantly, a systematic screen of local interneurons in the adult AL revealed unexpected cell type diversity and wiring variability of these neurons (Chou et al., 2010). In fact, from a total of
131 single cells analyzed in a GAL4 line that labels no more than seven neurons, 76 distinct glomerular innervation patterns were observed, suggesting that local interneurons are highly variable across individual flies. Our own observations extend this notion to the larval olfactory pathway by adding two pieces of evidence: the branching variability of types 2,4 , and $5 \mathrm{ALNs}$ in the AL and/or SOG and the erratic, nonclassifiable dendritic arborization patterns of wide-field PNs (Fig. 10B). Based on their detailed experimental approaches, Chou and co-workers (2010) proposed that primary features of neurons (commonly called "cell types" or "subtypes") may be genetically prespecified, whereas branching variability at more subtle levels may be due to cell-cell interactions during development or to neuronal activity and plasticity.

In any case, wiring variability appears to emerge as a second principle of insect brain organization, in parallel with stereotypy (Fig. 10A; Cachero and Jefferis, 2008). Consequently, the general significance of circuit models 
established from single or even a few individuals may be questionable. Also, variable connectivity should be taken into account in models of brain function, which so far were almost exclusively based on stereotypic connectivity. An imminent question is how differently wired brains accomplish common functions, such as translating sensory inputs into the same behavioral output. As one of the few suggestions addressing this issue, tiling of the entire adult AL by "patchy" local interneurons was suggested to be sufficient for proper signal processing, even if the patterns of tiling differ across flies (Chou et al., 2010). However, how different patterns of signal transfer in different brains are translated into a common function remains a mystery.

\section{Functional considerations}

What do our anatomical data suggest in terms of $\mathrm{AL}$ function? We show that $\alpha$-GABA staining overlaps with some of the neurons labeled by NP2426-GAL4 and NP3056-GAL4, extending previous findings with 189YGAL4 (Python and Stocker, 2002b). This suggests an inhibitory function of these neurons. In contrast, our attempt at identifying an excitatory, cholinergic class of ALNs similar to the adult AL (Olsen et al., 2007; Root et al., 2007; Shang et al., 2007) remained inconclusive, although cholinergic local interneurons were reported in the AL of first instar larvae (L. Prieto Godino, Cambridge UK, personal communication).

The arborization patterns of most types of ALNs we describe differ considerably within the AL, which may be related to specific aspects of odor processing. Type 1 ALNs share the AL innervation pattern of adult panglomerular local interneurons (Chou et al., 2010), suggesting similar functional properties. In contrast, type, 2, 3, 4, and $5 \mathrm{ALNs}$ branch predominantly or exclusively in subregions of the $A L$, such as the posterior half in subtype $2 \mathrm{~A}$ and type 4 ALNs, the lateral half in type 3 ALNs, the ventromedial half in type $5 \mathrm{ALNs}$, or a tiny medial $\mathrm{AL}$ region in subtype 2B ALNs. In this context we also note the often prominent innervation of glomerulus 1a, a putative target of aromatic odors (Asahina et al., 2009), by subtype 5A ALNs. Tentative mapping of larval odor ligands (Kreher et al., 2008; Asahina et al., 2009) in the AL suggested spatial heterogeneity of odor representation, for example, a clustering of glomeruli that potentially respond to aromatics in the lateral half of the AL (Masuda-Nakagawa et al., 2009). Links between heterogeneous ALN arborization patterns and the glomerular odor map are to be expected.

Perhaps the most intriguing issue regarding structurefunction relationships refers to the role of ALNs that interconnect the AL with the SOG, especially types 2, 3, 4, and 5 ALNs. Do the SOG arborizations of these neurons represent input or output sites, or both? Do they establish direct synaptic connectivity with taste afferents (Colomb et al., 2007) or with hugin cells, i.e., putative gustatory interneurons involved in feeding control (Melcher and Pankratz, 2005; Bader et al., 2007)? The broad areas of gustatory terminals and hugin neuron branches in the SOG indeed render such connectivity possible. However, for an unequivocal demonstration of direct synapses, more sophisticated approaches such as electron microscopy or GRASP (GFP reconstitution across synaptic partners; Gordon and Scott, 2009) would be required. Convergence of taste and odor circuits, wherever they occur in the brain, provides the anatomical substrate of tastereinforced olfactory learning, skills that have been demonstrated for adults as well as larvae (Thum et al., 2007; Selcho et al., 2009; Pauls et al., 2010a,b; for review, see Gerber et al., 2009). Accordingly, ALNs connecting the SOG and AL would represent ascending neurons that are involved in signal transfer toward higher olfactory centers. Neurons particularly suited for such function appear to be subtype $5 \mathrm{C} A L N s$, which relay the three major brain regions implicated in chemosensory learning, i.e., SOG, $\mathrm{AL}$, and MB. Alternatively, such neurons could be involved in feedback from the MB toward the AL and SOG. Indeed, functional evidence for descending neurons, although anatomically distinct, was provided for the adult olfactory circuit (Hu et al., 2010).

\section{Evolutionary links of circuit differences in the adult and larval antennal lobe}

Our data suggest that the patterns of horizontal connectivity are less well conserved between adult and larval ALs than the vertical circuitry provided by ORNs and PNs (Fig. 10). In fact, only subtype $1 \mathrm{~A}$ ALNs have their homologs in the adult AL (Das et al., 2008; Chou et al., 2010). Moreover, for most of the adult categories of local interneurons, including bilateral types, we were unable to identify larval equivalents. However, types of ALNs other than those described here may be revealed by using additional suitable GAL4 lines.

The most striking feature in the larval AL circuitry is the connectivity between the AL and the SOG that is provided by eight of the nine ALN subtypes. This implies special links in larvae between the two chemical senses smell and taste. This notion is supported by a number of other observations:

1. Whereas olfactory and gustatory sensilla in adults are strictly separated on different appendages, they are closely associated in the larval dorsal organ.

2. In terms of cell numbers, gustation dominates olfaction in larvae: they comprise an estimated 80 gustatory neurons but only 21 ORNs. In adults, which 
have $\approx 300$ gustatory neurons and $\approx 1,300$ ORNs, this relation is reversed.

3. Certain odorant receptors are also expressed in larval neurons that are not involved in olfactory function, neurons that target the SOG rather than the $\mathrm{AL}$ (Fishilevich et al., 2005; Kreher et al., 2005).

Do these differences in the design of adult and larval olfactory circuits reflect major discrepancies in chemosensory performance between flying and crawling stages? Obviously, adults vitally depend on long-range chemical signaling for food and mate recognition, whereas substrate-feeding larvae do not. Also, burrowing movements of the larva physically expose both gustatory and olfactory sensilla to the substrate. It is tempting to speculate that the relative importance of the two chemical senses and perhaps even the extent to which they can be distinguished differ between the two stages. Hence, the different patterns of connectivity in the adult and larval AL may only represent an anatomical correlate of the different chemosensory life styles.

\section{ACKNOWLEDGMENTS}

We thank the Kyoto and Bloomington stock centers, as well as L. Luo (Stanford University) and J. Simpson (HHMI, Janelia Farm Campus) for providing various fly strains. We are grateful to Alina von Essen and Dennis Pauls for discussion.

\section{LITERATURE CITED}

Asahina K, Louis M, Piccinotti S, Vosshall LB. 2009. A circuit supporting concentration-invariant odor perception in Drosophila. J Biol 8:9.

Bachy I, Retaux S. 2006. GABAergic specification in the basal forebrain is controlled by the LIM-hd factor Lhx7. Dev Biol 291:218-226.

Bader R, Colomb J, Pankratz B, Schröck A, Stocker RF, Pankratz MJ. 2007. Genetic dissection of neural circuit anatomy underlying feeding behavior in Drosophila: distinct classes of hugin expressing neurons. J Comp Neurol 502: 848-856.

Bhandawat V, Olsen SR, Gouwens NW, Schlief ML, Wilson RI. 2007. Sensory processing in the Drosophila antennal lobe increases reliability and separability of ensemble odor representations. Nat Neurosci 10:1474-1482.

Cachero S, Jefferis GSXE. 2008. Drosophila olfaction: the end of stereotypy? Neuron 59:843-845.

Chou YH, Spletter ML, Yaksi E, Leong JCS, Wilson RI, Luo L. 2010. Diversity and wiring variability of olfactory local interneurons in the Drosophila antennal lobe. Nat Neurosci 13:439-449.

Collins RT, Linker C, Lewis J. 2010. MAZe: a tool for mosaic analysis of gene function in zebrafish. Nat Methods 7:219-223.

Colomb J, Grillenzoni N, Ramaekers A, Stocker RF. 2007. Architecture of the primary taste center of Drosophila melanogaster larvae. J Comp Neurol 502:834-847.

Das A, Sen S, Lichtneckert R, Okada R, Ito K, Rodrigues V, Reichert H. 2008. Drosophila olfactory local interneurons and projection neurons derive from a common neuroblast lineage specified by the empty spiracles gene. Neural Dev 3:33.

Diegelmann S, Fiala A, Leibold C, Spall T, Buchner E. 2002. Transgenic flies expressing the fluorescence calcium sensor Cameleon 2.1 under UAS control. Genesis 34:95-98.

Fishilevich E, Domingos Al, Asahina K, Naef F, Vosshall LB, Louis M. 2005. Chemotaxis behavior mediated by single larval olfactory neurons in Drosophila. Curr Biol 15:2086-2096.

Gerber B, Stocker RF, Tanimura T, Thum AS. 2009. Smelling tasting, learning: Drosophila as a study case. In: Korsching S, Meyerhof W, editors. Chemosensory systems in mammals, fishes, and insects. Springer Review Series: Results and problems in cell differentiation. New York: Springer. p 139-185.

Gordon MD, Scott K. 2009. Motor control in a Drosophila taste circuit. Neuron 61:373-384.

Heimbeck G, Bugnon V, Gendre N, Keller A, Stocker RF. 2001. A central neural circuit for experience-independent olfactory and courtship behavior in Drosophila melanogaster. Proc Natl Acad Sci U S A 98:15336-15341.

Hu A, Zhang W, Wang Z. 2010. Functional feedback from mushroom bodies to antennal lobes in the Drosophila olfactory pathway. Proc Natl Acad Sci U S A 107: 10262-10267.

Ito K, Suzuki K, Estes P, Ramaswami M, Yamamoto D, Strausfeld NJ. 1998. The organization of extrinsic neurons and their implications in the functional roles of the mushroom bodies in Drosophila melanogaster Meigen. Learn Mem 5:52-77.

Jefferis GS, Hummel T. 2006. Wiring specificity in the olfactory system. Semin Cell Dev Biol 17:50-65.

Jefferis GS, Marin EC, Stocker RF, Luo L. 2001. Target neuron prespecification in the olfactory map of Drosophila. Nature 414:204-208

Jefferis GS, Potter CJ, Chan AM, Marin EC, Rohlfing T, Maurer CR Jr, Luo L. 2007. Comprehensive maps of Drosophila higher olfactory centers: spatially segregated fruit and pheromone representation. Cell 128:1187-1120.

Kim J, Chung YD, Park DY, Choi S, Shin DW, Soh H, Lee HW, Son W, Yim J, Park CS, et al. 2003. A TRPV family ion channel required for hearing in Drosophila. Nature 424:81-84.

Komiyama T, Luo L. 2006. Development of wiring specificity in the olfactory system. Curr Opin Neurobiol 16:67-73.

Komiyama T, Johnson WA, Luo L, Jefferis GS. 2003. From lineage to wiring specificity. POU domain transcription factors control precise connections of Drosophila olfactory projection neurons. Cell 24;112:157-67.

Krashes MJ, Keene AC, Leung B, Armstrong JD, Waddell S. 2007. Sequential use of mushroom body neuron subsets during Drosophila odor memory processing. Neuron 53: 103-115.

Kreher SA, Kwon JY, Carlson JR. 2005. The molecular basis of odor coding in the Drosophila larva. Neuron 46:445-456.

Kreher SA, Mathew D, Kim J, Carlson JR. 2008. Translation of sensory input into behavioral output via an olfactory system. Neuron 59:110-124.

Lahey T, Gorczyca M, Jia XX, Budnik V. 1994. The Drosophila tumor suppressor gene $d / g$ is required for normal synaptic bouton structure. Neuron 13:823-835.

Lai SL, Awasaki T, Ito K, Lee T. 2008. Clonal analysis of Drosophila antennal lobe neurons: diverse neuronal architectures in the lateral neuroblast lineage. Development 135: 2883-2893.

Larkin A, Karak S, Priva R, Das A, Ayyub C, Ito K, Rodrigues V, Ramaswami M. 2010. Central synaptic mechanisms underlie short-term olfactory habituation in Drosophila larvae. Learn Mem 17:645-653.

Lee T, Lee A, Luo L. 1999. Development of the Drosophila mushroom bodies: sequential generation of three distinct types of neurons from a neuroblast. Development 126:4065-4076. 
Marin EC, Jefferis GS, Komiyama T, Zhu H, Luo L. 2002. Representation of the glomerular olfactory map in the Drosophila brain. Cell 109:243-255.

Martin JR, Ernst R, Heisenberg M. 1998. Mushroom bodies suppress locomotor activity in Drosophila melanogaster. Learn Mem 5:179-191.

Masuda-Nakagawa LM, Tanaka NK, O'Kane CJ. 2005. Stereotypic and random patterns of connectivity in the larval mushroom body calyx of Drosophila. Proc Natl Acad Sci U S A 102:19027-19032.

Masuda-Nakagawa LM, Gendre N, O'Kane CJ, Stocker RF. 2009. Localized olfactory representation in mushroom bodies of Drosophila larvae. Proc Natl Acad Sci U S A 106:10314-10319.

Masuda-Nakagawa LM, Awasaki T, Ito K, O'Kane CJ. 2010. Targeting expression to projection neurons that innervate specific mushroom body calyx and antennal lobe glomeruli in larval Drosophila. Gene Expr Patterns 10:328-337.

Melcher C, Pankratz MJ. 2005. Candidate gustatory interneurons modulating feeding behavior in the Drosophila brain. PLoS Biol 3:e305.

Moreno N, Gonzalez A. 2007. Development of the vomeronasal amygdala in anuran amphibians: hodological, neurochemical, and gene expression characterization. J Comp Neurol 503:815-831.

Murthy M, Fiete I, Laurent G. 2008. Testing odor response stereotypy in the Drosophila mushroom body. Neuron 59: 1009-1023.

Ng M, Roorda RD, Lima SQ, Zemelman BV, Morcillo P, Miesenböck G. 2002. Transmission of olfactory information between three populations of neurons in the antennal lobe of the fly. Neuron 36:463-474.

Okada R, Awasaki T, Ito K. 2009. Gamma-aminobutyric acid (GABA)-mediated neural connections in the Drosophila antennal lobe. J Comp Neurol 514:74-91.

Olsen SR, Wilson RI. 2008. Lateral presynaptic inhibition mediates gain control in an olfactory circuit. Nature 452: 956-960.

Olsen SR, Bhandawat V, Wilson RI. 2007. Excitatory interactions between olfactory processing channels in the Drosophila antennal lobe. Neuron 54:89-103.

Osborne KA, Robichon A, Burgess E, Butland S, Shaw RA, Coulthard A, Pereira HS, Greenspan RJ, Sokolowski MB. 1997. Natural behavior polymorphism due to a cGMP-dependent protein kinase of Drosophila. Science 277:834-836.

Parnas D, Haghighi AP, Fetter RD, Kim SW, Goodman CS 2001. Regulation of postsynaptic structure and protein localization by the Rho-type guanine nucleotide exchange factor dPix. Neuron 32:415-424.

Pauls D, Pfitzenmaier JER, Krebs-Wheaton R, Selcho M Stocker RF, Thum AS. 2010a. Electric shock-induced associative olfactory learning in Drosophila larvae. Chem Senses 35:335-346.

Pauls D, Selcho M, Gendre N, Stocker RF, Thum AS. 2010b. Drosophila larvae establish appetitive olfactory memories via mushroom body neurons of embryonic origin. J Neurosci 30:10655-10666.

Python F, Stocker RF. 2002a. Adult-like complexity of the larval antennal lobe of $D$. melanogaster despite markedly low numbers of odorant receptor neurons. J Comp Neurol 445:374-387.

Python F, Stocker RF. 2002b. Immunoreactivity against choline acetyltransferase, $\gamma$-aminobutyric acid, histamine, octopamine, and serotonin in the larval chemosensory system of Drosophila. J Comp Neurol 453:157-167

Ramaekers A, Magnenat E, Marin EC, Gendre N, Jefferis GSXE, Luo L, Stocker RF. 2005. Glomerular maps without cellular redundancy at successive levels of the Drosophila larval olfactor circuit. Curr Biol 15:982-992.
Rodrigues V, Hummel T. 2008. Development of the Drosophila olfactory system. Adv Exp Med Biol 628:82-101.

Root CM, Semmelhack JL, Wong AM, Flores J, Wang JW. 2007. Propagation of olfactory information in Drosophila. Proc Natl Acad Sci U S A 104:11826-11831.

Seki Y, Rybak J, Wicher D, Sachse S, Hansson BS. 2010. Physiological and morphological characterization of local interneurons in the Drosophila antennal lobe. J Neurophysiol 104:1007-19

Selcho M, Pauls D, Han KA, Stocker RF, Thum AS. 2009. The role of dopamine in Drosophila larval classical olfactory conditioning. PLoS One 4:e5897.

Shang Y, Claridge-Chang A, Sjulson L, Pypaert M, Miesenböck G 2007. Excitatory local circuits and their implications for olfactory processing in the fly antennal lobe. Cell 128:601-612.

Spletter ML, Liu J, Liu J, Su H, Giniger E, Komiyama T, Quake S, Luo L. 2007. Lola regulates Drosophila olfactory projection neuron identity and targeting specificity. Neural Dev $16 ; 2: 14$

Stocker RF, Heimbeck G, Gendre N, de Belle JS. 1997. Neuroblast ablation in Drosophila P[GAL4] lines reveals origins of olfactory interneurons. J Neurobiol 32:443-456.

Takagawa K, Salvaterra P. 1996. Analysis of choline acetyltransferase protein in temperature sensitive mutant flies using newly generated monoclonal antibody. Neurosci Res 24:237-243.

Tanaka NK, Tanimoto H, Ito K 2008. Neuronal assemblies of the Drosophila mushroom body. J Comp Neurol 10;508: 711-55.

Tanaka NK, Ito K, Stopfer M. 2009. Odor-evoked neural oscillations in Drosophila are mediated by widely branching interneurons. J Neurosci 29:8595-8603.

Thorne N, Chromey C, Bray S, Amrein H. 2004. Taste perception and coding in Drosophila. Curr Biol 14:1065-1079.

Thum AS, Knapek S, Rister J, Dierichs-Schmitt E, Heisenberg M, Tanimoto H. 2006. Differential potencies of effector genes in adult Drosophila. J Comp Neurol 498:194-203.

Thum AS, Jenett A, Ito K, Heisenberg M, Tanimoto H. 2007. Multiple memory traces for olfactory reward learning in Drosophila. J Neurosci 27:11132-11138.

Vosshall LB, Stocker RF. 2007. Molecular architecture of smell and taste in Drosophila. Annu Rev Neurosci 30 505-533.

Wang J, Ma X, Yang JS, Zheng X, Zugates CT, Lee $\mathrm{CH}$, Lee T. 2004a. Transmembrane/ juxtamembrane domain-dependent Dscam distribution and function during mushroom body neuronal morphogenesis. Neuron 43:663-672.

Wang JW, Wong AM, Flores J, Vosshall LB, Axel R. 2003. An olfactory sensory map in the fly brain. Cell 102:271-282.

Wang Z, Singhvi A, Kong P, Scott K. 2004b. Taste representations in the Drosophila brain. Cell 117:981-991.

Whiteland JL, Nicholls SM, Shimeld C, Easty DC, Williams NA, Hill TJ. 1995. Immunohistochemical detection of T-cell subsets and other leukocytes in paraffin-embedded rat and mouse tissues with monoclonal antibodies. J Histochem Cytochem 43:313-320.

Wilson RI, Laurent G. 2005. Role of GABAergic inhibition in shaping odor-evoked spatiotemporal patterns in the Drosophila antennal lobe. J Neurosci 25:9069-9079.

Wilson RI, Turner GC, Laurent G. 2004. Transformation of olfactory representations in the Drosophila antennal lobe. Science 303:366-370.

Wong AM, Wang JW, Axel R. 2002. Spatial representation of the glomerular map in the Drosophila protocerebrum. Cell 109:229-241.

Yang MY, Armstrong JD, Vilinsky I, Strausfeld NJ, Kaiser K. 1995. Subdivision of the Drosophila mushroom bodies by enhancer-trap expression patterns. Neuron 15:45-54. 